\title{
Correlates of protection against symptomatic and asymptomatic SARS-CoV-2 infection
}

\author{
Shuo Feng1, Daniel J. Phillips $\mathbb{1}^{1}$, Thomas White ${ }^{2}$, Homesh Sayal ${ }^{2}$, Parvinder K. Aley', Sagida Bibi', \\ Christina Dold', Michelle Fuskova $\mathbb{D}^{3}$, Sarah C. Gilbert $\mathbb{D}^{3}$, Ian Hirsch ${ }^{2}$, Holly E. Humphries ${ }^{4}$, \\ Brett Jepson ${ }^{5,6}$, Elizabeth J. Kelly7, Emma Plested', Kathryn Shoemaker5, Kelly M. Thomas ${ }^{4}$, \\ Johan Vekemans ${ }^{8}$, Tonya L. Villafana ${ }^{5}$, Teresa Lambe ${ }^{109,35}$, Andrew J. Pollard ${ }^{1,10,35}$, \\ Merryn Voysey ${ }^{1,10,35 \bowtie}$ and the Oxford COVID Vaccine Trial Group*
}

\begin{abstract}
The global supply of COVID-19 vaccines remains limited. An understanding of the immune response that is predictive of protection could facilitate rapid licensure of new vaccines. Data from a randomized efficacy trial of the ChAdOx1 nCoV-19 (AZD1222) vaccine in the United Kingdom was analyzed to determine the antibody levels associated with protection against SARS-CoV-2. Binding and neutralizing antibodies at 28 days after the second dose were measured in infected and noninfected vaccine recipients. Higher levels of all immune markers were correlated with a reduced risk of symptomatic infection. A vaccine efficacy of $80 \%$ against symptomatic infection with majority Alpha (B.1.1.7) variant of SARS-CoV-2 was achieved with 264 (95\% Cl: 108, 806) binding antibody units (BAU)/ml: and 506 (95\% Cl: 135, not computed (beyond data range) (NC)) BAU/ml for anti-spike and anti-RBD antibodies, and 26 (95\% Cl: NC, NC) international unit (IU)/ml and 247 (95\% Cl: 101, NC) normalized neutralization titers $\left(\mathbf{N F}_{50}\right)$ for pseudovirus and live-virus neutralization, respectively. Immune markers were not correlated with asymptomatic infections at the $5 \%$ significance level. These data can be used to bridge to new populations using validated assays, and allow extrapolation of efficacy estimates to new COVID-19 vaccines.
\end{abstract}

W ithin 17 months of the identification of SARS-CoV-2 in Wuhan, China, in response to the pandemic, 6 COVID19 vaccines were recommended for use by the World Health Organization (WHO) as of 16 June 2021 (ref. ${ }^{1}$ ). Vaccine efficacies (VE) ranging from $50 \%$ to $95 \%$ against symptomatic COVID-19 infections have been reported, using varying endpoint definition $s^{2-7}$. Real-world evidence from vaccine-rollout programs has shown that COVID-19 vaccines are highly effective against severe disease, hospitalization, and death, and reduce both asymptomatic infection and within household transmission ${ }^{8-13}$.

The global supply of COVID-19 vaccines remains limited despite intense production efforts. Authorization of new vaccines could help meet demand. As more countries implement vaccine programs, it will become increasingly difficult to conduct clinical efficacy studies of new vaccines. Understanding the relationship between immune responses to vaccines and protection against clinical outcomes is urgently needed to speed vaccine development. Knowledge of immune measures that are statistically associated with protection against disease ('correlates of protection') may allow new vaccines to be authorized for use on the basis of immunogenicity and safety data alone, when large efficacy trials are not feasible. In addition, understanding the immune response allows for comparison of vaccines across cohorts of people who differ by age, race, ethnicity, or other factors.
Both binding and neutralizing antibodies are thought to be potential correlates of protection against COVID-19 and are correlated with each other ${ }^{3,14-16}$. Previous human challenge studies of seasonal coronaviruses reported high levels of baseline neutralizing antibodies in uninfected or asymptomatic people ${ }^{17}$. However, protection from infection with seasonal coronaviruses is not long-lasting ${ }^{17,18}$.

Early evidence from a fishery-vessel outbreak of SARS-CoV-2 suggested that higher levels of pre-existing neutralizing antibodies were potential correlates of protection ${ }^{18,19}$. A longitudinal cohort study of healthcare workers highlighted the association between baseline anti-spike and anti-nucleocapsid immunoglobulin $\mathrm{G}(\mathrm{IgG})$ and decreased risk of SARS-CoV-2 infection in the following 6 months ${ }^{19,20}$.

Evidence that antibodies may play a role in mediating protection against overt disease has come from vaccination and challenge studies in animals. Both neutralizing antibody titers and Fc-dependent functional antibody responses correlate with protection induced by DNA and adenoviral vector vaccines in rhesus macaques (Macaca mulatta) ${ }^{21,22}$. Additionally, higher doses of passively transferred monoclonal antibodies were more protective than were lower doses in golden hamsters (Mesocricetus auratus) and rhesus macaques challenged with the SARS-CoV-2 virus containing D614 in its spike protein ${ }^{23}$.

\footnotetext{
'Oxford Vaccine Group, Department of Paediatrics, University of Oxford, Oxford, UK. '2Late-stage development, Respiratory and Immunology (R\&I), BioPharmaceuticals R\&D, AstraZeneca, Cambridge, UK. ${ }^{3}$ The Jenner Institute, Nuffield Department of Medicine, University of Oxford, Oxford, UK. ${ }^{4}$ National Infection Service, Public Health England, Salisbury, UK. ${ }^{5}$ Late-stage development, Respiratory and Immunology (R\&I), BioPharmaceuticals R\&D, AstraZeneca, Gaithersburg, MD, USA. ${ }^{6}$ Cytel Inc., Cambridge, MA, USA. ${ }^{7}$ Microbial Sciences, BioPharmaceuticals R\&D, AstraZeneca, Gaithersburg, MD, USA. ${ }^{8}$ Late-stage development Respiratory and Immunology (R\&I), BioPharmaceuticals R\&D, AstraZeneca, Gothenburg, Sweden. ${ }^{9} \mathrm{Chinese}$ Academy of Medical Science (CAMS) Oxford Institute (COI), University of Oxford, Oxford, UK. ${ }^{10}$ NIHR Oxford Biomedical Centre, Oxford, UK. ${ }^{35}$ These authors contributed equally: Teresa Lambe, Andrew J. Pollard, Merryn Voysey. ${ }^{\star} \mathrm{A}$ list of authors and their affiliations appears at the end of the paper. 凶e-mail: merryn.voysey@paediatrics.ox.ac.uk
} 
Table 1 | Baseline characteristics of correlates population, control population, and cases and noncases among correlates cohort

ChAdOx1 nCoV-19

correlates population $(n=$

4,372)

\section{MenACWY control \\ population $(n=4,194)$}

ChAdOx1 nCoV-19 correlates cohort ${ }^{\mathrm{a}}$

Cases $(n=171)$

Noncases $(n=1,404)$

\section{Age group}

18-55 years

56-69 years

$\geq 70$ years

Sex (Female)

Ethnicity

White

Asian

Black

Other ${ }^{b}$

BMI (mean (s.d.))

$\mathrm{BMI}<30$

$\mathrm{BMI} \geq 30$

Comorbidities

Respiratory disease

Cardiovascular disease

Diabetes

Healthcare worker status

Nonhealthcare worker

Healthcare worker facing no more than one patient with COVID-19 per day

Healthcare worker facing at least one patient $816(18.7 \%)$

with COVID-19 per day

Baseline risk probabilities ${ }^{c}$

Mean (s.d.)

Dosage schedule

LD/LD

LD/SD

SD/SD

Prime-boost interval

$<6$ weeks

6-8 weeks

9-11 weeks

$\geq 12$ weeks

Length of follow-up (days) from 7 days post PB28 until infection occurred or Feb 282021 (median (IQR))

\begin{tabular}{|ll}
\hline NAAT+ cases & 174 \\
\hline Symptomatic & $55(31.6 \%)$ \\
\hline Asymptomatic & $99(56.9 \%)$ \\
\hline Nonprimary symptomatic & $20(11.5 \%)$ \\
\hline
\end{tabular}

$0.0786(0.0303)$

\begin{tabular}{l}
$3,240(74.1 \%)$ \\
$542(12.4 \%)$ \\
$590(13.5 \%)$ \\
$2,533(57.9 \%)$ \\
\hline $4,036(92.3 \%)$ \\
$220(5.0 \%)$ \\
$21(0.5 \%)$ \\
$95(2.2 \%)$ \\
\hline $26.4(5)$ \\
\hline $3,519(80.5 \%)$ \\
$852(19.5 \%)$ \\
\hline $1,088(24.9 \%)$ \\
\hline $547(12.5 \%)$ \\
\hline $572(13.1 \%)$ \\
\hline $99(2.3 \%)$ \\
\hline
\end{tabular}

$1,652(37.8 \%)$

$1,904(43.6 \%)$

125 (2.9\%)

$1,420(32.5 \%)$

$1,078(24.7 \%)$
$538(12.3 \%)$
$1,158(26.5 \%)$
$1,598(36.6 \%)$
$88(64,113)$

$2,827(64.7 \%)$

$3,229(77 \%)$
$482(11.5 \%)$
$483(11.5 \%)$
$2,526(60.2 \%)$

$3,914(93.3 \%)$

$184(4.4 \%)$

$15(0.4 \%)$

$81(1.9 \%)$

$26.5(5.2)$

3,347 (79.8\%)

$846(20.2 \%)$

$1,032(24.6 \%)$

$537(12.8 \%)$

$514(12.3 \%)$

85 (2\%)

$1,456(34.7 \%)$

$1,938(46.2 \%)$

$800(19.1 \%)$

$0.0794(0.0296)$

$69(1.6 \%)$

$1,361(32.5 \%)$

2,764 (65.9\%)

$931(22.2 \%)$
$478(11.4 \%)$
$1,236(29.5 \%)$
$1,549(36.9 \%)$
$85(62,108)$

333

196 (58.9\%)

112 (33.6\%)

$25(7.5 \%)$
$144(84.2 \%)$
$10(5.8 \%)$
$17(9.9 \%)$
$102(59.6 \%)$

$160(93.6 \%)$

8 (4.7\%)

$1(0.6 \%)$

$2(1.2 \%)$

27 (5.2)

$130(76.0 \%)$

$41(24.0 \%)$

$44(25.7 \%)$

$20(11.7 \%)$

24 (14.0\%)

$3(1.8 \%)$

65 (38.0\%)

74 (43.3\%)

$32(18.7 \%)$

$0.0824(0.0283)$

$0.0774(0.0306)$

BMI, body mass index; LD, low dose; SD, standard dose ${ }^{2}$ The correlates cohort is a subset of all eligible participants in the ChAdOx1 nCoV-19 correlates populations who have samples processed for at least one assay. 'Options included in 'Other' are as follows: 'Mixed', 'Other - Free text', or 'prefers not to give'. 'The baseline risk exposure score summarizes predicted probability of having NAAT+ outcome from the risk model developed using the MenACWY Control Population.

A meta-analysis modeling the relationship between VE reported from phase 3 vaccine clinical trials and neutralization titers in convalescent patients showed a significant association at the study level between VE and neutralizing antibody levels ${ }^{24}$. Nevertheless, no study to date has defined a correlate of protection against SARS-CoV-2 infection or disease that can be used by regulators and vaccine developers. 

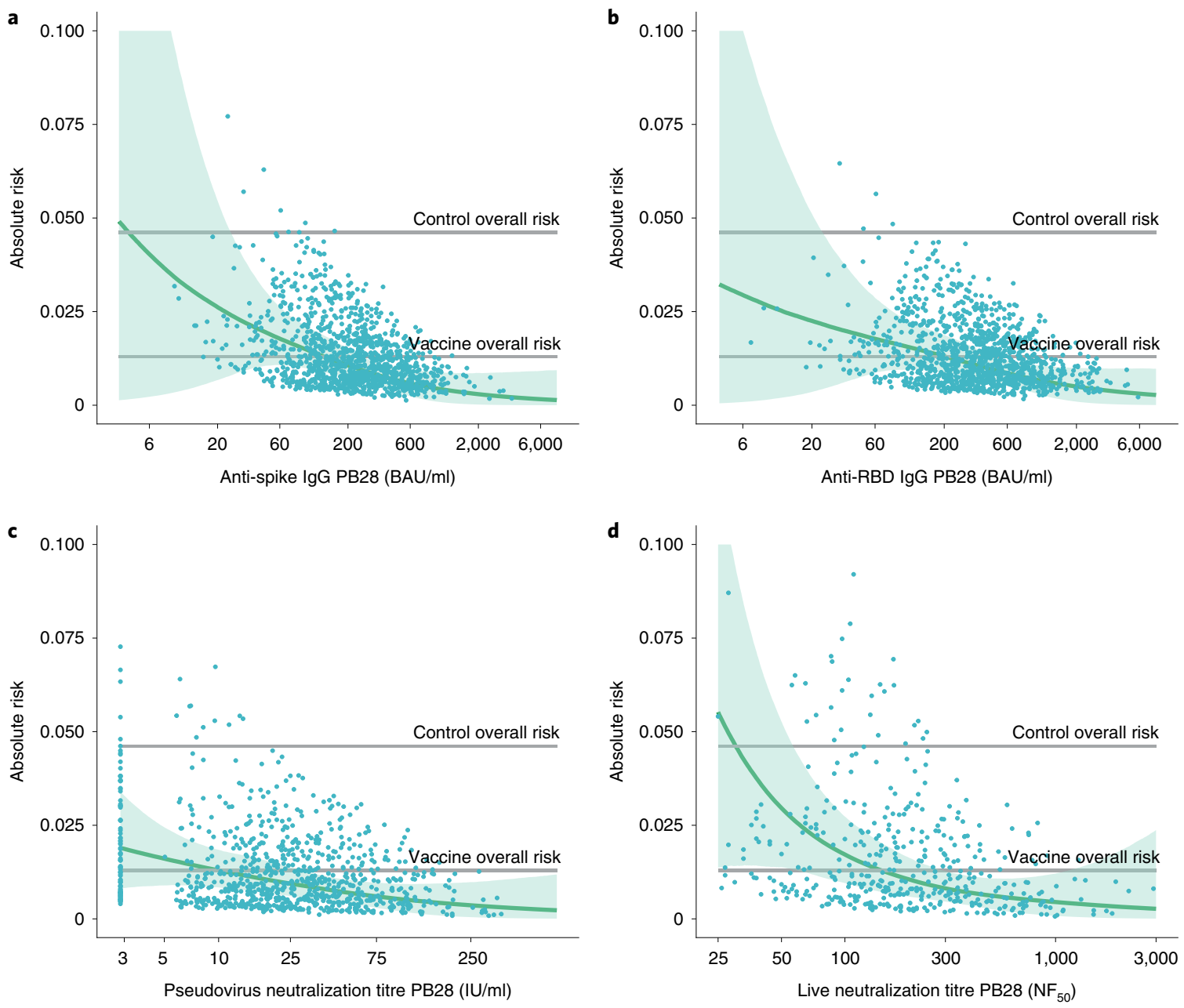

Fig. 1 | Predicted absolute risk of primary symptomatic COVID-19 as a function of immune markers measured at PB28 by generalized additive regression. a-d, Predicted absolute risk of primary symptomatic COVID-19 as a function of anti-spike lgG measured at PB28 (52 cases, 1,155 noncases included in the analysis) (a), anti-RBD lgG measured at PB28 (52 cases, 1,155 noncases included in the analysis) (b), pseudovirus neutralization antibody titers at PB28 (47 cases, 828 noncases included in the analysis (c), and live-virus neutralization antibody titers PB28 (36 cases, 412 noncases included in the analysis) (d). Gray horizontal lines show the overall risk of primary symptomatic COVID-19 in the control group (MenACWY) and vaccine groups (ChAdOx1 nCoV-19). Blue dots show the absolute risk predicted from the model across the range of antibody values included in the analysis, adjusting for baseline exposure risk to SARS-CoV-2 infection. Green shaded areas show the $\mathrm{Cl}$ around the predicted mean probability (green line).

The ChAdOx1 nCoV-19 vaccine (AZD1222) is a chimpanzee adenoviral vector vaccine with full-length SARS-CoV-2 spike insert which was developed at the University of Oxford and is in widespread global use and produced by AstraZeneca and their manufacturing partners. Using data from the United Kingdom and Brazil, we previously estimated an overall VE of $66.7 \%$ (95\% confidence interval (CI): 57.4 to 74.0 ) against symptomatic infection and $27.3 \%$ (95\% CI: -17.2 to 54.9$)$ against asymptomatic infection ${ }^{2,3}$. We previously showed that estimates of VE against symptomatic COVID-19 infection were higher in subgroups with higher pseudovirus neutralization antibody titers, or higher anti-spike IgG levels, in vaccine clinical trials of ChAdOx1 nCoV19 in adults using summarized data ${ }^{3}$. Here, we report the relationship between a continuous measure of the humoral immune responses to vaccination and protection afforded by this vaccine, which may facilitate further vaccine development. Specifically, we used individual data from the United Kingdom and identified the thresholds for four immune markers associated with protection against symptomatic infection. The WHO international standard units are reported for all assays, to allow comparisons across studies and platforms.

\section{Results}

Using the COV002 data from the United Kingdom, we assessed the correlation between immune markers at 28 days post the second dose (post-boost +28 days, PB28) of ChAdOx1 nCoV-19 and symptomatic and asymptomatic infections. Participants were reminded weekly to contact their study site if they experienced any of the primary symptoms of COVID- 19 (fever $\geq 37.8^{\circ} \mathrm{C}$; cough; shortness of breath; anosmia or ageusia) and were assessed in clinic with a nose and throat swab taken for nucleic acid amplification testing (NAAT). Additionally, participants were asked to complete a nose and throat swab at home each week, which was used to detect asymptomatic infections. Nucleic acid amplification test positive (NAAT+) participants who had symptoms other than the main five COVID-19 symptoms were categorized as nonprimary symptomatic and were not included in correlates analysis.

Table 1 summarizes baseline characteristics for the defined correlates population, control population, and correlates cohort by status (cases and noncases). Extended Data Fig. 1 summarizes the exclusions for each study group. Participants were followed for a median of 88 and 85 days, counting from 7 days after the PB28 visit, among correlates and control populations, respectively. 
a

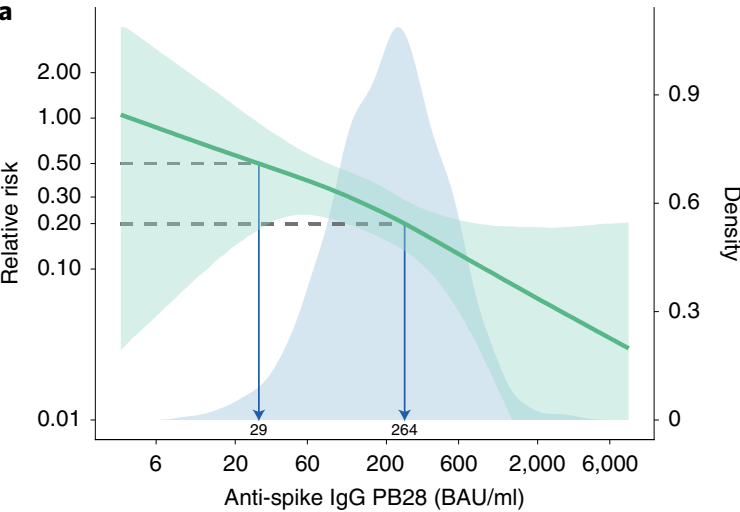

c

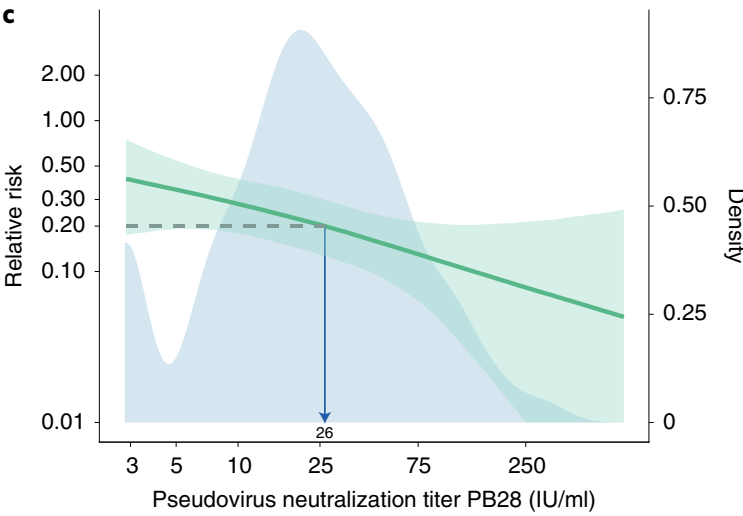

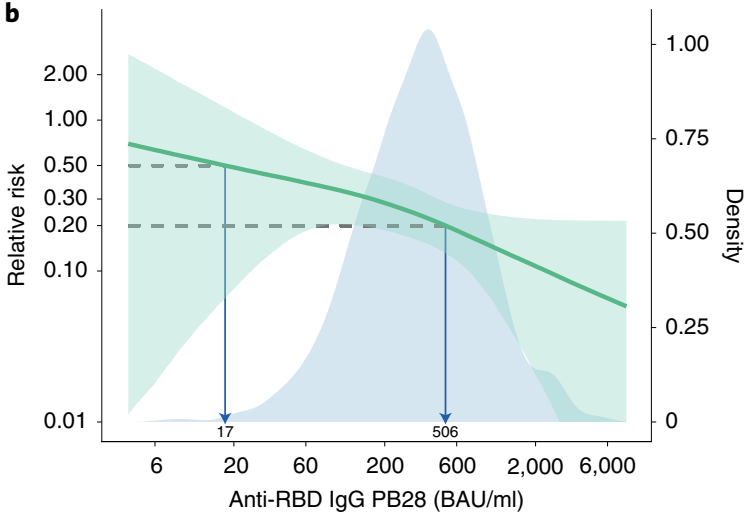

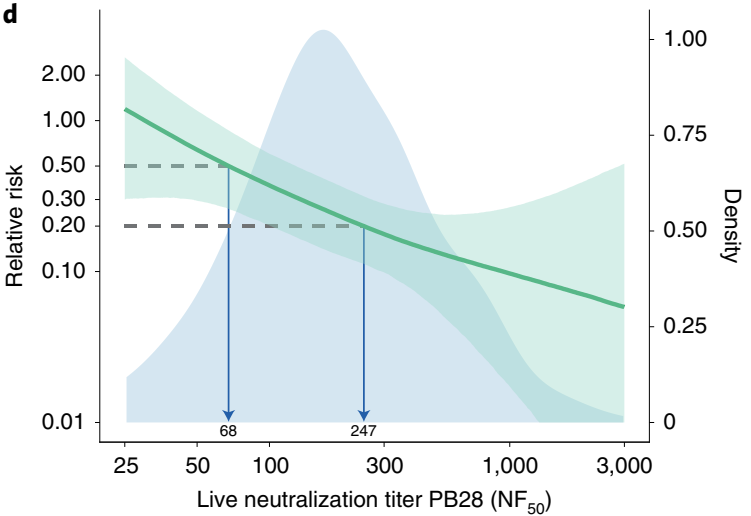

Fig. 2 | Relative risk of primary symptomatic COVID-19 among vaccine recipients compared with MenACWY control arm participants as a function of immune markers measured at PB28. a, Anti-spike IgG measured at PB28 (52 cases, 1,155 noncases included in the analysis). b, Anti-RBD IgG measured at PB28 (52 cases, 1,155 noncases included in the analysis). c, Pseudovirus neutralization antibody titers at PB28 (47 cases, 828 noncases included in the analysis). d, Live-virus neutralization antibody titers at PB28 (36 cases, 412 noncases included in the analysis). Blue shaded areas represent the immune marker density distribution. Green lines show the relative risk of infection among vaccine recipients compared with that of the MenACWY control arm participants, derived by dividing the output curve from Fig. 1 by the overall risk of infection in the MenACWY control group. The green lines are the median relative risk obtained from 10,000 bootstrap samples. Green shaded areas are $95 \%$ bootstrapped $\mathrm{Cls}$ for the relative risk. The arrows point to the immune marker values at 0.20 and 0.50 relative risk, that is $80 \%$ and $50 \%$ VE for illustrative purpose. The full range of VE estimates from 50 to $90 \%$ are shown in Table 2.

The follow-up time was censored at the earliest timing of infection, withdrawal, or unblinding, or the cut-off date 28 February 2021. Among 4,372 participants in the correlates population, there were a total of 174 breakthrough NAAT+ cases of SARS-CoV-2 infection. Data were available for at least one of four assay readouts (anti-spike IgG, anti-receptor binding domain (RBD) IgG, pseudovirus neutralization assay, and live-virus neutralization) for 171 out of $174(98.3 \%)$ cases and 1,404 out of 4,195 (33.5\%) noncases. Data were available for anti-spike and anti-RBD IgG from 1,318 PB28 samples (163 cases and 1,155 noncases, Supplementary Table 2). A smaller set of data was available for analysis for pseudovirus neutralization titers (149 cases, 828 noncases) and for live-virus neutralization (110 cases and 412 noncases) (Supplementary Table 2). People in the case group were younger, with $84.2 \%$ being aged $18-55$ years, compared with $71.6 \%$ of the noncase group, and were more likely to be healthcare workers $(62.0 \%$ were healthcare workers compared with $57.5 \%$ of the noncase group, Table 1). In our baseline exposure model developed among the MenACWY control group, younger age and being a healthcare worker facing more than one patient with COVID-19 per day were associated with a higher risk of being NAAT + . Other variables were not significant (see model output in Supplementary Table 3). The distribution of baseline risk was similar for cases and noncases (Table 1).
Antibody levels at PB28 in cases and noncases across four immune markers are shown in Extended Data Fig. 2. Anti-spike IgG and anti-RBD IgG were highly correlated with each other (Pearson correlation coefficient $r=0.926$ ), while the correlation between pseudovirus neutralization titer and normalized live-virus neutralization titer $\left(\mathrm{NF}_{50}\right)$ was moderate $(r=0.572)$. Anti-spike IgG values were also correlated with pseudovirus neutralization titers $(r=0.657)$ and normalized live-virus neutralization titers $\left(\mathrm{NF}_{50}\right)$ $(r=0.600)$ (Extended Data Fig. 3). Non-normalized live-virus neutralization titers $\left(\mathrm{ND}_{50}\right)$ were less highly correlated with anti-spike $\operatorname{IgG}(r=0.411)$ and pseudovirus neutralization titers $(r=0.305)$.

The risk of symptomatic COVID-19 decreased with increasing levels of anti-spike $\operatorname{IgG}(P=0.003)$, anti-RBD $\operatorname{IgG}(P=0.018)$, pseudovirus neutralization titer $(P=0.005)$, and live-virus neutralization titer $(P<0.001)$ (Figs. 1 and 2 and Table 2$)$. In contrast, there were no significant associations between any of the assays and protection against asymptomatic infection including for sensitivity analysis restricting to high viral load (all $P>0.05$, Fig. 3, Extended Data Figs. 4, and 5, and Supplementary Table 4). When primary symptomatic COVID-19 cases were classified according to the presence of shortness of breath, we observed a similar trend, with increasing immune marker levels associated with lower risk of infection (all $P<0.05$, Supplementary Table 4 and Extended Data Fig. 6), but not for those with no shortness of breath (all $P>0.05$, 


\begin{tabular}{|c|c|c|c|c|c|c|c|c|c|}
\hline Assay units & $P$ value $_{\text {immune marker }}$ & $P$ value $_{\text {baseline risk score }}$ & $\begin{array}{l}\text { No. } \\
\text { cases }\end{array}$ & $\begin{array}{l}\text { No. } \\
\text { noncase }\end{array}$ & $\begin{array}{l}50 \% \text { VE } \\
(95 \% \mathrm{Cl})\end{array}$ & $\begin{array}{l}60 \% \text { VE } \\
(95 \% \mathrm{Cl})\end{array}$ & $\begin{array}{l}70 \% \text { VE } \\
(95 \% \mathrm{Cl})\end{array}$ & $\begin{array}{l}80 \% \text { VE } \\
(95 \% \mathrm{Cl})\end{array}$ & $\begin{array}{l}90 \% \text { VE } \\
(95 \% \mathrm{Cl})\end{array}$ \\
\hline \multicolumn{10}{|c|}{ Anti-spike IgG } \\
\hline $\mathrm{AU} / \mathrm{ml}$ & 0.003 & $<0.001$ & 52 & 1155 & $\begin{array}{l}4446 \\
(N C, 12822)\end{array}$ & $\begin{array}{l}8413 \\
(N C, 22232)\end{array}$ & $\begin{array}{l}17538 \\
(N C, 37929)\end{array}$ & $\begin{array}{l}40923(16748 \\
125017)\end{array}$ & $\begin{array}{l}139306 \\
(57276, N C)\end{array}$ \\
\hline \multicolumn{10}{|c|}{ Anti-RBD IgG } \\
\hline $\mathrm{AU} / \mathrm{ml}$ & 0.018 & $<0.001$ & 52 & 1155 & $\begin{array}{l}2193 \\
(N C, 13614)\end{array}$ & $\begin{array}{l}6266 \\
(N C, 29105)\end{array}$ & $\begin{array}{l}20700 \\
(N C, 56620)\end{array}$ & $\begin{array}{l}63383 \\
(16903, N C)\end{array}$ & $\begin{array}{l}295781 \\
(90567, N C)\end{array}$ \\
\hline $\mathrm{BAU} / \mathrm{ml}$ & & & & & $17(\mathrm{NC}, 109)$ & $50(N C, 232)$ & $\begin{array}{l}165 \\
(N C, 452)\end{array}$ & $506(135, N C)$ & $\begin{array}{l}2360 \\
(723, N C)\end{array}$ \\
\hline $\mathrm{ID}_{50}$ & 0.005 & $<0.001$ & 47 & 828 & NC & $22(N C, 76)$ & $57(N C, 183)$ & $185(\mathrm{NC}, \mathrm{NC})$ & $982(303, N C)$ \\
\hline $\mathrm{IU} / \mathrm{ml}$ & & & & & NC & $3(N C, 11)$ & $8(N C, 26)$ & $26(N C, N C)$ & $140(43, N C)$ \\
\hline
\end{tabular}

$\mathrm{ID}_{50}$ neutralization dilution for $50 \%$ virus inhibition; NC: not computed; $\mathrm{AU} / \mathrm{ml}$ : arbitrary units per $\mathrm{mL} ; \mathrm{BAU} / \mathrm{ml}$ : binding antibody units per $\mathrm{ml}$ (WHO international standard $20 / 136$ ), IU/ml: international units per $\mathrm{ml}$ (WHO international standard 20/136). Where Cls were outside the range of values of the assay the limits are reported as NC. VE estimates and Cls are those shown in Fig. 4 , at every $10 \%$ increment in the $y$ axis. The two-sided $P$ value for each immune marker (column 2 ) is from the generalized additive models in Fig. 1, showing the strength of the relationship between the antibody value and infection. The $P$ values were not adjusted for multiple comparisons.

Supplementary Table 4 and Extended Data Fig. 7). Higher pseudovirus and live-virus neutralization titers were associated with lower risk of infection for those who had three or more COVID-19 symptoms (Supplementary Table 4 and Extended Data Fig. 8). The number of cases and noncases included for correlates analysis by each immune marker and outcome has been summarized in Table 2 and Supplementary Table 4.

The antibody level associated with $80 \%$ VE against primary symptomatic COVID-19 was 40,923 (95\% CI: $16,748,125,017)$ arbitrary units $(\mathrm{AU}) / \mathrm{ml}$ for anti-spike IgG, equivalent to $264 \mathrm{BAU} / \mathrm{ml}$ (95\% CI: 108, 806) using the WHO international standard (NIBSC code $20 / 136)$. For anti-RBD IgG, $80 \%$ efficacy was achieved with median antibody level of 506 (95\% CI: 135, not computed (NC)) BAU/ml (Figs. 2 and 4 and Table 2).

For pseudovirus and live-virus neutralizing antibody titers, values of 26 (95\% CI: NC, NC) IU/ml and 247 (95\% CI: 101, NC) normalized neutralization titers $\left(\mathrm{NF}_{50}\right)$, respectively, were associated with $80 \%$ VE against symptomatic infection (Table 2). No values from these assays were associated with protection against asymptomatic infection (Supplementary Table 4)

For all assays, when the analysis was restricted to symptomatic cases with shortness of breath, $80 \% \mathrm{VE}$ was achieved at lower levels of immune markers than for symptomatic cases in general. Higher baseline exposure risk of SARS-CoV-2 infections predict higher probability of all outcomes (all $P<0.05$, Table 2 and Supplementary Table 4$)$, except for asymptomatic infections $(P>0.05)$ in generalized additive models.

\section{Discussion}

Here, we report an analysis of potential correlates of protection using data from 171 cases of SARS-CoV-2 infection and 1,404 noncases, showing that higher anti-spike IgG, anti-RBD IgG, and neutralizing antibody titers are all associated with lower risk of symptomatic disease. We used immune responses in a phase $2 / 3$ clinical trial to derive a model to predict absolute risk of infection, with appropriate adjustment for bias, assigning estimates for each level of antibody in the dataset. The relative risk of infection was then derived by reference to risk of infection in the control group. This is a robust approach to derive population estimates and was adapted from recently described methods ${ }^{25,26}$.

We previously published overall aggregate-level summaries of antibody levels in participants with different prime-boost intervals. Vaccination prime-boost intervals were associated with varying levels of $\mathrm{VE}$, and there are some intriguing similarities between the aggregate-level data with the estimate provided from analysis of individual participant level data in this report. The estimated anti-spike IgG level of $40,923 \mathrm{AU} / \mathrm{ml}$ and the pseudovirus neutralizing antibody titer of 185 associated with $80 \%$ VE in our models were similar to the geometric mean titers of $48,961 \mathrm{AU} / \mathrm{ml}$ and 237.0, respectively, previously reported in the subgroup of participants vaccinated with ChAdOx1 nCoV-19 with a dose interval of at least 12 weeks between their first and second dose-a regimen that provided $80.0 \%$ (95\% CI 65.2 to 88.5 ) VE in the pooled analysis of clinical trial data from the United Kingdom, Brazil, and South Africa $^{3}$. The aggregate-level results previously published included all eligible participants in the assessment of VE, but only those with available antibody data were included in the summaries of immunogenicity, meaning that direct comparisons of efficacy with immunogenicity were not in the same populations. Our current approach analyzes the relationship between infections and antibody levels at the individual level in a single set of participants, with appropriate adjustment for confounding, providing robust outputs. In addition, the current work provides outputs in WHO standard units, which are necessary for comparisons with data from other laboratories with different assays.

In a preprint by Gilbert et al., correlates of protection derived from the Moderna phase 3 efficacy trial are reported using similar methodology $y^{27}$. Although overall binding and pseudovirus neutralizing antibody titers after vaccination were higher in that study than those measured after the ChAdOx1 nCoV-19 vaccine, the correlates of protection findings appear similar to those we report here. 

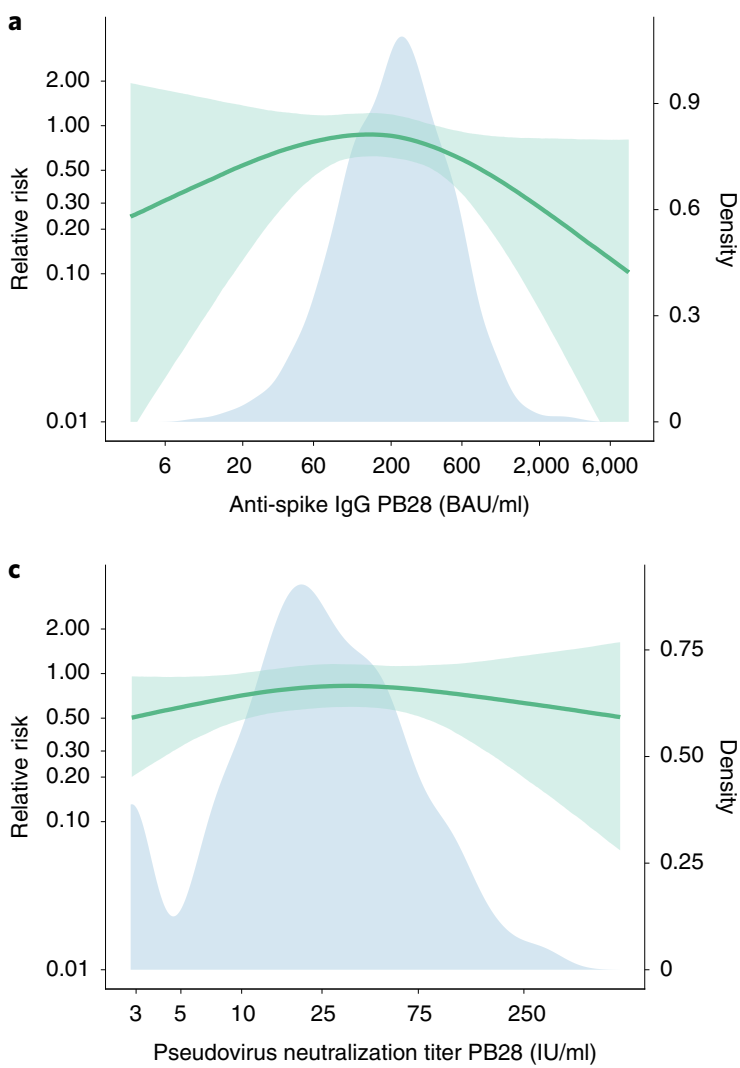
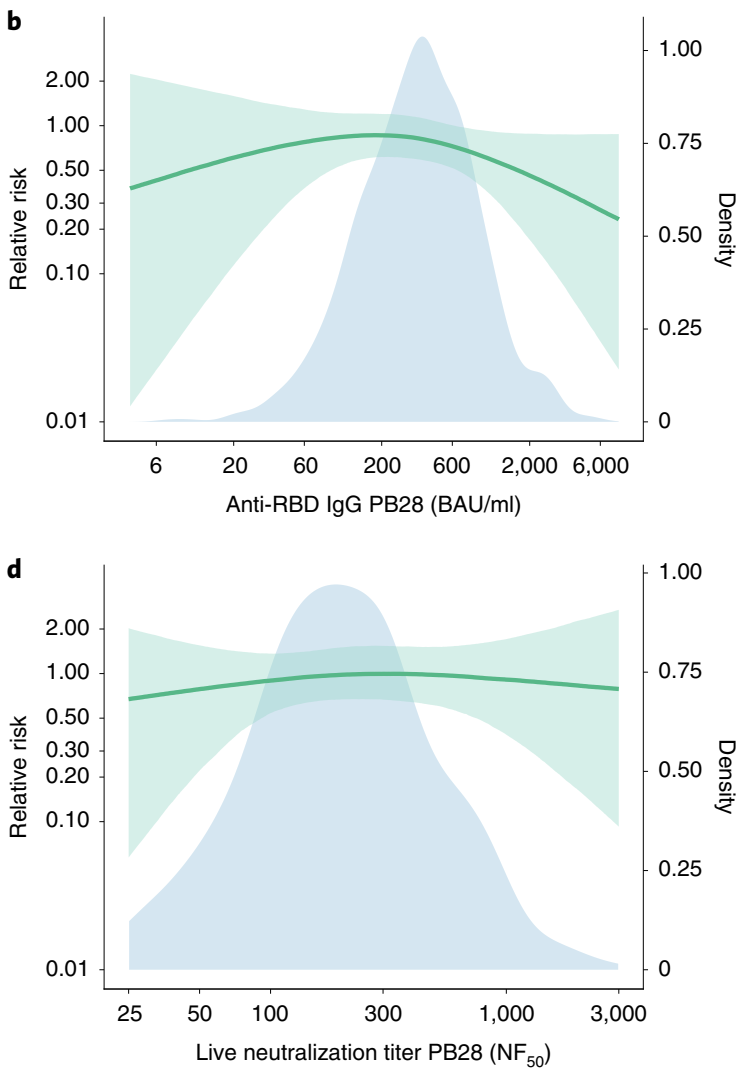

Fig. 3 | Relative risk of asymptomatic SARS-CoV-2 infection among vaccine recipients compared with the MenACWY control arm participants as a function of immune markers measured at PB28. a, Anti-spike lgG measured at PB28 ( 91 cases, 1,155 noncases included in the analysis). b, Anti-RBD IgG measured at PB28 (91 cases, 1,155 noncases included in the analysis). c, Pseudovirus neutralization antibody titers at PB28 (86 cases, 828 noncases included in the analysis). d, Live-virus neutralization antibody titers at PB28 (62 cases, 412 noncases included in the analysis). Blue shaded areas represent the immune marker density distribution. Green lines show the relative risk of infection among vaccine recipients compared with the MenACWY control arm participants. The green lines are the median relative risk obtained from 10,000 bootstrap samples. Green shaded areas are bootstrapped $95 \% \mathrm{Cls}$.

No serological measurements in our data were shown to correlate with protection against asymptomatic infection or against symptomatic illness with only mild upper respiratory symptoms. This is consistent with our interim analysis that VE against asymptomatic infection was $27.3 \%$ (95\% CI: -17.2 to 54.9 ) and was not significant at the $5 \%$ level $^{2}$. These results are consistent with the real-world observation that infection remains possible in fully vaccinated individuals, despite high effectiveness against severe disease.

The antibody correlates presented in this report relate to protection against mild disease, defined as a NAAT + test with at least one symptom present. Weekly self-swabbing in the trial enabled detection of many mild cases. At these antibody titers, efficacy against more severe endpoints, used in other trials, would be higher than the estimates in this analysis. Notably, this has been confirmed in the analysis of real-world effectiveness, in which the milder cases are not detected, after two doses of the vaccines in older adults in England, where VE was $90 \%$ for Pfizer and $89 \%$ for ChAdOx1 $\mathrm{nCoV}-19$ against symptomatic disease using the same case definition for both vaccines ${ }^{11}$, while lower efficacy estimates were measured in our previously reported efficacy analysis with a milder disease endpoint ${ }^{2}$.

The correlates of vaccine efficacy reported here could be used to extrapolate efficacy to immunogenicity data for novel vaccines where clinical efficacy results are unavailable. A trial of a new vaccine that works through similar immune mechanisms and which produces antibody responses that are above the correlate values reported here in at least $50 \%$ of participants (that is, it has a similar or higher median), might be expected to have similar efficacy against the clinical endpoints used in our UK trial, and higher efficacy against more severe endpoints. We provide correlates for vaccine efficacy estimates ranging from $50 \%$ to $90 \%$ to allow flexibility in the way these estimates are utilized by the regulators and policy-makers.

It has previously been shown that protection against lower respiratory tract infection (LRTI) may be easier to achieve than against upper respiratory tract infection (URTI), as challenge studies in rhesus macaques have shown stronger correlation between neutralizing titers and the level of subgenomic messenger RNA in bronchoalveolar lavage samples than in nasal swab samples ${ }^{28}$.

Similarly, ChAdOx1 nCoV-19-vaccinated hamsters, with low neutralizing titers against B.1.351, were fully protected against LRTI following challenge with B.1.351, despite no evidence of protection against shedding of virus from the upper airway ${ }^{29}$. Protection against upper respiratory tract or asymptomatic infections may be more closely associated with the presence of secretory IgA on the mucosal surface which was not measured in this study ${ }^{30}$.

These observations indicate that the reduced neutralizing capacity against B.1.351 and other variants of concern, might drive reduced protection against initial infection, and perhaps transmission, but protection against severe disease is maintained. Clinical trials of SARS-CoV-2 vaccines have consistently shown higher efficacy against more severe forms of disease, such as those causing hospitalization or death, than against mild infections $\mathbf{s}^{2-5,15,31}$. We are unable to assess correlates of protection against severe disease or hospitalization as there were no vaccinated participants hospitalized in the COV002 study. 

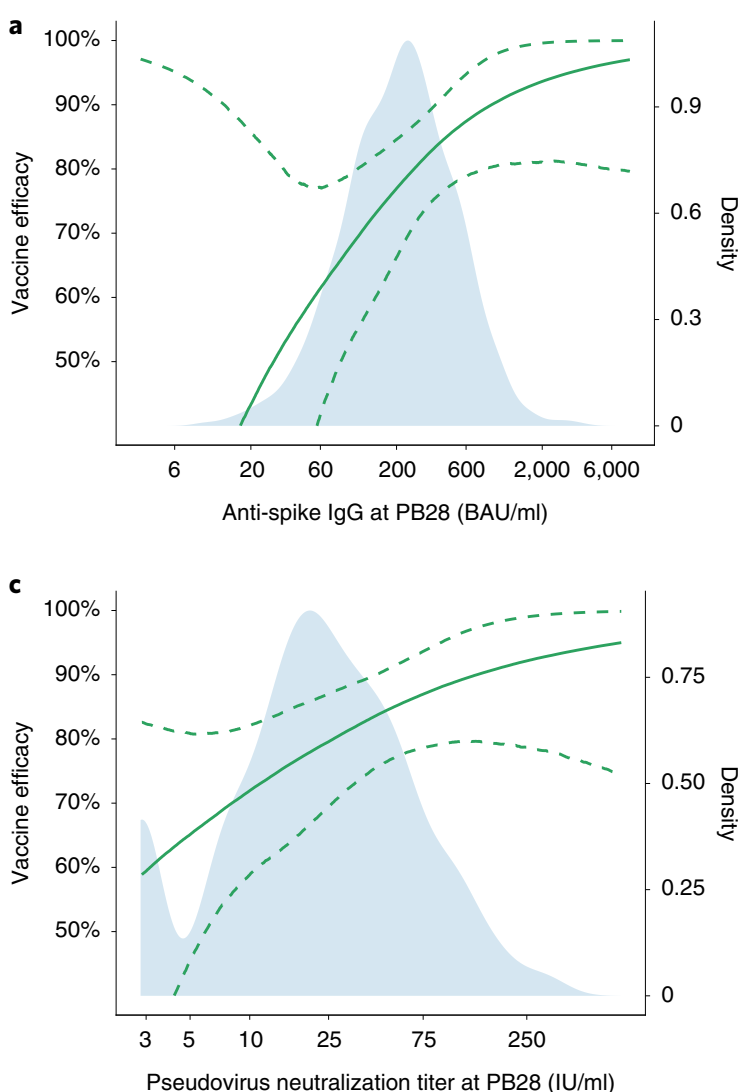

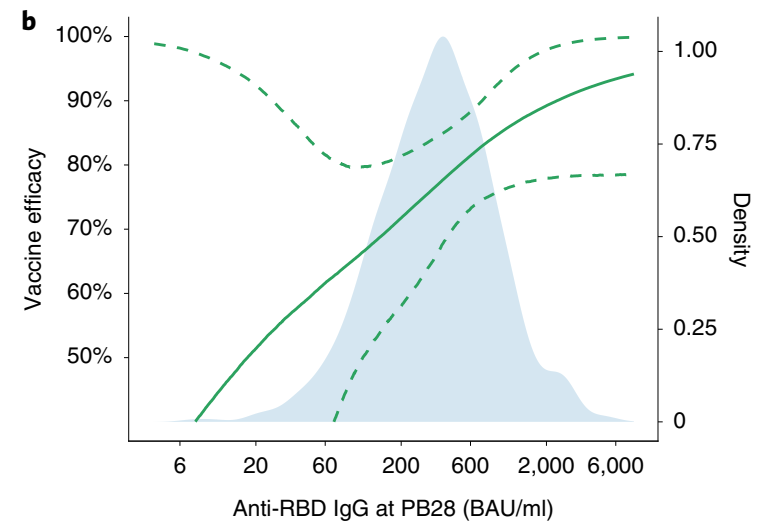

d

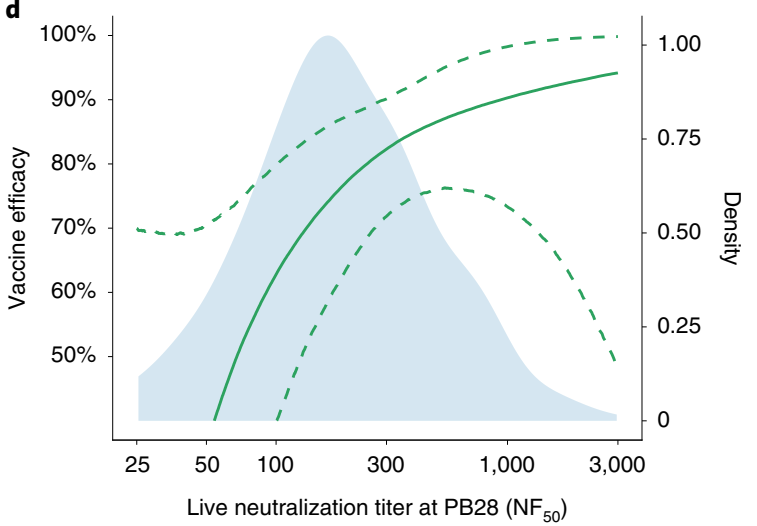

Fig. 4 | VE against primary symptomatic COVID-19 as a function of immune markers measured at PB28. a, Anti-spike lgG measured at PB28 (52 cases, 1,155 noncases included in the analysis). b, Anti-RBD IgG measured at PB28 (52 cases, 1,155 noncases included in the analysis). c, Pseudovirus neutralization antibody titers at PB28 (47 cases, 828 noncases included in the analysis). d, Live-virus neutralization antibody titers at PB28 (36 cases, 412 noncases included in the analysis). Blue shaded areas represent the immune marker density distribution. Green lines show the VE, and green dotted lines are $95 \%$ bootstrapped confidence intervals for VE. VE is computed as 1 minus the relative risks shown in Fig. 2. These results are also shown in Table 2 at $10 \%$ increments on the $y$ axis.

Although live-virus and pseudovirus neutralization assays were modestly correlated with each other, the live-virus assay was more closely associated with protection against symptomatic COVID-19 than was the pseudovirus assay. This may reflect the sensitivity and dynamic range of the assays.

Protection against symptomatic COVID-19 is not absolute with any vaccine, and the results presented here show that there is no single threshold value for any of the assays investigated that was indicative of sterilizing immunity in our data. Instead, the probability of infection decreases on average with higher immune responses, but substantial variation exists between individuals. This is similar to studies of respiratory syncytial virus, in which risk of infection decreased with higher antibody levels, although infections were still observed at high antibody levels, suggesting that a definitive individual threshold of protection does not exist ${ }^{32}$. We provide antibody estimates that correspond with $50 \%$ to $90 \%$ VE; however, the wide CIs around these estimates should be noted.

These estimates represent the antibody level observed 28 days after a second dose of vaccine that provide protection during the subsequent 4- to 6-month period among UK COV002 efficacy and immunogenicity cohorts. This is different from the antibody level that would protect an individual at the time of exposure to the virus. Further work is needed to determine the durability of antibody and long-term protection after vaccination.

High levels of protection were noted after vaccination with one dose of a lipid nanoparticle RNA vaccine, despite modest levels of neutralizing antibody, strongly supporting the concept that other mechanisms are at play as co-correlates of protection ${ }^{5,33}$. We have previously shown that a wide range of Fc-mediated antibody functions are induced by vaccination, and it is possible that these functions may be important in the absence of neutralizing antibody ${ }^{34}$. Furthermore, strong T cell responses induced by ChAdOx1 nCoV19 may contribute to protection ${ }^{14,16}$ and have been associated with recovery from COVID-19 disease ${ }^{35-37}$. The relationship between antibody and $\mathrm{T}$ cell responses may differ depending on the type of vaccine used, and care should be taken when interpreting data from clinical testing of different vaccine technologies.

There are some limitations to our analysis. These analyses are based on cases of COVID-19 detected in a mainly white population in the United Kingdom, which were mostly due to B.1.177 and B.1.1.7 variants. In settings in which these are not the dominant variants causing disease, or where neutralization assays use different strains of the virus, the modeled relationships between immune markers and disease outcomes shown here may not apply. In addition, we have conducted a large number of analyses, and therefore some caution should be taken when drawing conclusions on the basis of single $P$ values alone as these are presented unadjusted for multiple comparisons. Furthermore, these analyses have been conducted on samples taken after two doses of ChAdOx1 nCoV19 and might not apply to protection afforded by a single dose of the same vaccine or other COVID-19 vaccines. Correlates may also vary according to age profile, but this was not explored in our study due to the small number of older adults recruited. The potential role of $\mathrm{T}$ cells and interaction between humoral and cellular immunity 
has not been evaluated in this study. It is not possible to determine in this study if our results represent mechanistic or nonmechanistic correlates of protection, as many immune responses are highly correlated.

Correlates of protection can be used to bridge to new populations and new vaccines using validated assays. These data can be used to extrapolate efficacy estimates for new vaccines that use similar immune mechanisms and where efficacy data is unavailable.

\section{Online content}

Any methods, additional references, Nature Research reporting summaries, source data, extended data, supplementary information, acknowledgements, peer review information; details of author contributions and competing interests; and statements of data and code availability are available at https://doi.org/10.1038/ s41591-021-01540-1.

Received: 21 June 2021; Accepted: 14 September 2021; Published online: 29 September 2021

\section{References}

1. World Health Organisation. The COVID-19 candidate vaccine landscape. https://www.who.int/publications/m/item/draft-landscape-of-covid19-candidate-vaccines (2021)

2. Voysey, M. et al. Safety and efficacy of the ChAdOx $1 \mathrm{nCoV}-19$ vaccine (AZD1222) against SARS-CoV-2: an interim analysis of four randomised controlled trials in Brazil, South Africa, and the UK. Lancet 397, 99-111 (2021).

3. Voysey, M. et al. Single-dose administration and the influence of the timing of the booster dose on immunogenicity and efficacy of ChAdOx1 nCoV-19 (AZD1222) vaccine: a pooled analysis of four randomised trials. Lancet 397 , 881-891 (2021).

4. Moderna. Moderna's COVID-19 vaccine candidate meets its primary efficacy endpoint in the first interim analysis of the phase 3 COVE study. https:// investors.modernatx.com/news-releases/news-release-details/modernas-covid19-vaccine-candidate-meets-its-primary-efficacy (2020).

5. Polack, F. P. et al. Safety and efficacy of the BNT162b2 mRNA Covid-19 vaccine. N. Engl. J. Med. 383, 2603-2615 (2020).

6. Centers for Disease Control and Prevention. Johnson \& Johnson's Janssen COVID-19 vaccine overview and safety. https://www.cdc.gov/ coronavirus/2019-ncov/vaccines/different-vaccines/janssen.html (2021).

7. Al Kaabi, N. et al. Effect of 2 inactivated SARS-CoV-2 vaccines on symptomatic COVID-19 infection in adults: a randomized clinical trial. JAMA 326, 35-45 (2021).

8. Tenforde, M. W. et al. Effectiveness of Pfizer-BioNTech and Moderna vaccines against COVID-19 among hospitalized adults aged $\geq 65$ years United States, January-March 2021. MMWR Morb. Mortal. Wkly Rep. 70, 674-679 (2021).

9. Haas, E. J. et al. Impact and effectiveness of mRNA BNT162b2 vaccine against SARS-CoV-2 infections and COVID-19 cases, hospitalisations, and deaths following a nationwide vaccination campaign in Israel: an observational study using national surveillance data. Lancet 397, 1819-1829 (2021).

10. Vasileiou, E., et al. Effectiveness of first dose of COVID-19 vaccines against hospital admissions in Scotland: national prospective cohort study of 5.4 million people. in SSRN (2021)

11. Public Health England. COVID-19 vaccine surveillance report Week 20. https://assets.publishing.service.gov.uk/government/uploads/system/uploads/ attachment_data/file/988193/Vaccine_surveillance_report_-_week_20.pdf (2021).

12. Lopez Bernal, J. et al. Effectiveness of the Pfizer-BioNTech and OxfordAstraZeneca vaccines on COVID-19 related symptoms, hospital admissions, and mortality in older adults in England: test negative case-control study. Brit. Med. J. 373, n1088 (2021).

13. Pritchard, E. et al. Impact of vaccination on new SARS-CoV-2 infections in the United Kingdom. Nat. Med. 27, 1370-1378 (2021).

14. Folegatti, P. M. et al. Safety and immunogenicity of the ChAdOx1 nCoV-19 vaccine against SARS-CoV-2: a preliminary report of a phase $1 / 2$, single-blind, randomized controlled trial. Lancet 396, 467-478 (2020).

15. Emary, K. R. W. et al. Efficacy of ChAdOx1 nCoV-19 (AZD1222) vaccine against SARS-CoV-2 variant of concern 202012/01 (B.1.1.7): an exploratory analysis of a randomised controlled trial. Lancet 397, 1351-1362 (2021).
16. Ewer, K. J. et al. T cell and antibody responses induced by a single dose of ChAdOx1 nCoV-19 (AZD1222) vaccine in a phase 1/2 clinical trial. Nat. Med. 27, 270-278 (2020).

17. Callow, K. A. Effect of specific humoral immunity and some non-specific factors on resistance of volunteers to respiratory coronavirus infection. J. Hyg. 95, 173-189 (1985)

18. Edridge, A. W. D. et al. Seasonal coronavirus protective immunity is short-lasting. Nat. Med. 26, 1691-1693 (2020).

19. Addetia, A. et al. Neutralizing antibodies correlate with protection from SARS-CoV-2 in humans during a fishery vessel outbreak with a high attack rate. J. Clin. Microbiol. 58, e02107-e02120 (2020).

20. Lumley, S. F. et al. Antibody status and incidence of SARS-CoV-2 infection in health care workers. N. Engl. J. Med. 384, 533-540 (2021).

21. $\mathrm{Yu}$, J. et al. DNA vaccine protection against SARS-CoV-2 in rhesus macaques. Science 369, 806-811 (2020).

22. Mercado, N. B. et al. Single-shot Ad26 vaccine protects against SARS-CoV-2 in rhesus macaques. Nature 586, 583-588 (2020).

23. Baum, A. et al. REGN-COV2 antibodies prevent and treat SARS-CoV-2 infection in rhesus macaques and hamsters. Science 370, 1110-1115 (2020).

24. Khoury, D. S. et al. Neutralizing antibody levels are highly predictive of immune protection from symptomatic SARS-CoV-2 infection. Nat. Med. 27, 1205-1211 (2021).

25. Qin, L., Gilbert, P. B., Corey, L., McElrath, M. J. \& Self, S. G. A framework for assessing immunological correlates of protection in vaccine trials. J. Infect. Dis. 196, 1304-1312 (2007).

26. USG COVID-19 Response Team / Coronavirus Prevention Network (CoVPN) Biostatistics Team. Vaccine efficacy trial immune correlates statistical analysis plan. https://figshare.com/articles/online resource/ CoVPN_OWS_COVID-19_Vaccine_Efficacy_Trial_Immune_Correlates_ SAP/13198595/11 (2021)

27. Gilbert, P. B., et al. Immune correlates analysis of the mRNA-1273 COVID-19 vaccine efficacy trial. Preprint at medRxiv https://doi org/10.1101/2021.08.09.21261290 (2021).

28. van Doremalen, N. et al. ChAdOx1 nCoV-19 vaccine prevents SARS-CoV-2 pneumonia in rhesus macaques. Nature 586, 578-582 (2020).

29. Fischer, R. J., et al. ChAdOx1 nCoV-19 (AZD1222) protects hamsters against SARS-CoV-2 B.1.351 and B.1.1.7 disease. Nature Comm. (in the press).

30. Wang, Z. et al. Enhanced SARS-CoV-2 neutralization by dimeric IgA. Sci. Transl. Med. 13, eabf1555 (2021).

31. Kemp, A. AZD1222 US Phase III trial met primary efficacy endpoint in preventing COVID-19 at interim analysis. https://www.astrazeneca.com/ media-centre/press-releases/2021/ astrazeneca-us-vaccine-trial-met-primary-endpoint.html (2021).

32. Walsh, E. \& Falsey, A. R. Humoral and mucosal immunity in protection from natural respiratory syncytial virus infection in adults. J. Infect. Dis. 190, 373-378 (2004)

33. Mulligan, M. J. et al. Phase I/II study of COVID-19 RNA vaccine BNT162b1 in adults. Nature 586, 589-593 (2020).

34. Barrett, J. R. et al. Phase $1 / 2$ trial of SARS-CoV-2 vaccine ChAdOx1 nCoV-19 with a booster dose induces multifunctional antibody responses. Nat. Med. 27, 279-288 (2020).

35. McMahan, K. et al. Correlates of protection against SARS-CoV-2 in rhesus macaques. Nature 590, 630-634 (2021)

36. Yang, P.-H. et al. Increased circulating level of interleukin- 6 and $\mathrm{CD} 8^{+} \mathrm{T}$ cell exhaustion are associated with progression of COVID-19. Infect. Dis. Poverty 9, $161(2020)$.

37. Urra, J. M., Cabrera, C. M., Porras, L. \& Ródenas, I. Selective CD8 cell reduction by SARS-CoV-2 is associated with a worse prognosis and systemic inflammation in COVID-19 patients. Clin. Immunol. 217, 108486-108486 (2020).

Publisher's note Springer Nature remains neutral with regard to jurisdictional claims in published maps and institutional affiliations.

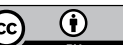

Open Access This article is licensed under a Creative Commons Attribution 4.0 International License, which permits use, sharing, adaptation, distribution and reproduction in any medium or format, as long as you give appropriate credit to the original author(s) and the source, provide a link to the Creative Commons license, and indicate if changes were made. The images or other third party material in this article are included in the article's Creative Commons license, unless indicated otherwise in a credit line to the material. If material is not included in the article's Creative Commons license and your intended use is not permitted by statutory regulation or exceeds the permitted use, you will need to obtain permission directly from the copyright holder. To view a copy of this license, visit http://creativecommons. org/licenses/by/4.0/.

(c) The Author(s) 2021 


\section{the Oxford COVID Vaccine Trial Group}

Syed Adlou', Lauren Allen ${ }^{4}$, Brian Angus ${ }^{3}$, Rachel Anslow', Marie-Claude Asselin ${ }^{4}$, Natalie Baker ${ }^{4}$, Philip Baker', Thomas Barlow ${ }^{4}$, Amy Beveridge', Kevin R. Bewley ${ }^{4}$, Phillip Brown ${ }^{4}$, Emily Brunt ${ }^{4}$, Karen R. Buttigieg ${ }^{4}$, Susana Camara', Sue Charlton ${ }^{4}$, Emily Chiplin ${ }^{4}$, Paola Cicconi ${ }^{3}$, Elizabeth A. Clutterbuck ${ }^{1}$, Andrea M. Collins ${ }^{1,12}$, Naomi S. Coombes ${ }^{4}$, Sue Ann Costa Clemens ${ }^{1,13}$, Melanie Davison ${ }^{4}$, Tesfaye Demissie', Tanya Dinesh', Alexander D. Douglas ${ }^{3}$, Christopher J. A. Duncan ${ }^{14,15}$, Katherine R. W. Emary', Katie J. Ewer' ${ }^{3}$, Sally Felle', Daniela M. Ferreira", Adam Finn ${ }^{16,77}$, Pedro M. Folegatti ${ }^{3}$, Ross Fothergill ${ }^{4}$, Sara Fraser ${ }^{4}$, Harriet Garlant ${ }^{4}$, Laura Gatcombe ${ }^{4}$, Kerry J. Godwin ${ }^{4}$, Anna L. Goodmann ${ }^{18,19}$, Christopher A. Green ${ }^{20,21}$, Bassam Hallis ${ }^{4}$, Thomas C. Hart ${ }^{1}$,

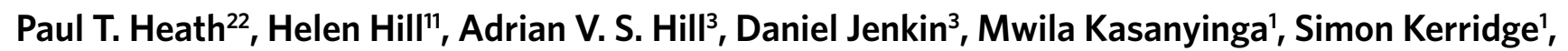
Chanice Knight ${ }^{4}$, Stephanie Leung ${ }^{4}$, Vincenzo Libri ${ }^{23,24}$, Patrick J. Lillie ${ }^{25}$, Spyridoula Marinou', Joanna McGlashan ${ }^{4}$, Alastair C. McGregor ${ }^{26,27}$, Lorna McInroy ${ }^{4}$, Angela M. Minassian' ${ }^{3}$, Yama F. Mujadidi', Elizabeth J. Penn ${ }^{4}$, Christos J. Petropoulos ${ }^{28}$, Katrina M. Pollock ${ }^{29}$, Pamela C. Proud ${ }^{4}$, Samuel Provstgaard-Morys', Durga Rajapaska', Maheshi N. Ramasamy', Katherine Sanders', Imam Shaik ${ }^{4}$, Nisha Singh ${ }^{1}$, Andrew Smith ${ }^{30}$, Matthew D. Snape', Rinn Song', Sonu Shrestha', Rebecca K. Sutherland ${ }^{31}$, Emma C. Thomson ${ }^{32}$, David P. J. Turner ${ }^{33}$, Alice Webb-Bridges', Terri Wrin ${ }^{28}$ and Christopher J. Williams ${ }^{34}$

\footnotetext{
${ }^{11}$ Department of Clinical Sciences, Liverpool School of Tropical Medicine, Liverpool, UK. ${ }^{12}$ Liverpool University Hospitals NHS Foundation Trust, Liverpool, UK. ${ }^{13}$ Institute of Global Health, University of Siena, Siena, Italy. ${ }^{14}$ Department of Infection and Tropical Medicine, Newcastle upon Tyne Hospitals NHS Foundation Trust, Newcastle, UK. ${ }^{15}$ Translational and Clinical Research Institute, Immunity and Inflammation Theme, Newcastle University, Newcastle, UK. ${ }^{16}$ School of Population Health Sciences, University of Bristol, Bristol, UK. ${ }^{7}$ University Hospitals Bristol and Weston NHS Foundation Trust, Bristol, UK. ${ }^{18}$ Department of Infectious Diseases, Guy's and St Thomas' NHS Foundation Trust, St Thomas' Hospital, London, UK. ${ }^{19}$ MRC Clinical Trials Unit at University College London, London, UK. ${ }^{20}$ NIHR/Wellcome Trust Clinical Research Facility, University Hospitals Birmingham NHS Foundation Trust, Birmingham, UK. ${ }^{21}$ Institute of Microbiology \& Infection, University of Birmingham, Birmingham, UK. ${ }^{22}$ St George's Vaccine Institute, St George's, University of London, London, UK. ${ }^{23}$ NIHR UCLH Clinical Research Facility, London, UK. ${ }^{24} \mathrm{NIHR}$ UCLH Biomedical Research Centre, London, UK. ${ }^{25} \mathrm{Hull}$ University Teaching Hospitals NHS Trust and Hull York Medical School, Hull, UK. ${ }^{26}$ London North West University Healthcare NHS Trust, London, UK. ${ }^{27}$ Department of Medicine, Imperial College London, London, UK. ${ }^{28}$ Labcorp-Monogram Biosciences, South San Francisco, CA, USA. ${ }^{29}$ NIHR Imperial Clinical Research Facility and NIHR Imperial Biomedical Research Centre, London, UK. ${ }^{30}$ College of Medical, Veterinary \& Life Sciences, Glasgow Dental Hospital \& School, University of Glasgow, Glasgow, UK. ${ }^{31}$ Clinical Infection Research Group, Regional Infectious Diseases Unit, NHS Lothian, Edinburgh, UK. ${ }^{32}$ MRC University of Glasgow Centre for Virus Research \& Department of Infectious Diseases, Queen Elizabeth University Hospital, Glasgow, UK. ${ }^{33}$ University of Nottingham and Nottingham University Hospitals NHS Trust, Nottingham, UK. ${ }^{34}$ Aneurin Bevan University Health Board, Newport, UK.
} 


\section{Methods}

Study description. The data included in this analysis comes from participants enrolled in COV002 (registration NCT04400838), a phase 2/3 randomized single-blind vaccine efficacy trial conducted across 19 sites in the United Kingdom. A full description of the trial including immunogenicity, efficacy, and safety data, and the protocol has been previously published ${ }^{2,3,14,15,38}$

This study was approved in the United Kingdom by the Medicines and Healthcare products Regulatory Agency (MHRA), reference 21584/0428/001 0001, and the South-Central Berkshire Research Ethics Committee, reference 20/ SC/0179. All participants provided informed consent.

Briefly, participants in the study were randomized to receive ChAdOx1 nCoV-19 or a MenACWY control vaccine. The randomization ratio (ChAdOx1 nCoV-19:MenACWY) differed by study cohort, and was either 1:1, 5:1, or 3:1. (see CONSORT diagram, Extended Data Fig. 1). Open label groups are not included in this report.

Study endpoints and outcomes. Participants were reminded weekly to contact their study site if they experienced any of the primary symptoms of COVID-19 (fever $\geq 37.8^{\circ} \mathrm{C}$; cough; shortness of breath; anosmia or ageusia) and were assessed in clinic, with a nose and throat swab taken for NAAT. In addition, participants were asked to complete a nose and throat swab at home each week.

The outcomes for this analysis were (1) primary symptomatic COVID-19, that is a NAAT + swab with at least one qualifying symptom, and (2) asymptomatic infections identified from weekly self-administered swabs, defined as a NAAT+ swab with no symptom reported. Sensitivity analysis of asymptomatic infections removed potential false-positive cases by restricting to those with higher viral load (cycle threshold $(\mathrm{CT})$ value $<30$ ). NAAT+ participants who had symptoms other than the main five COVID-19 symptoms were categorized as nonprimary symptomatic and were not included in correlates analysis.

Primary symptomatic COVID-19 outcomes were further classified according to whether a symptomatic participant reported shortness of breath or not, and whether three or more COVID-19 symptoms among five were present, indicators of more severe disease.

All endpoints were evaluated by a blinded independent clinical review committee.

Immune markers and time points. A proportion of serum samples from vaccine recipients at PB28 were tested on 3 different assays with 4 assay readouts. All NAAT + cases were tested if sample volume allowed, and a proportion of noncases were tested. Samples were tested blinded to case status. The data from noncases were obtained first, and consisted mainly of the samples processed for the initial application for emergency use which needed $15 \%$ of samples included in the efficacy cohort to be processed on validated assays. Subsequent to this NAAT+ cases were sent for testing as they occurred, if not already including the $15 \%$. We assume the mechanism of missingness for samples that were not tested to be missing at random ${ }^{39}$. To account for the missing data, factors associated with sample availability were controlled as weights in the analysis (see 'Correlates of risk' and 'Inverse probability weighting' below)

Anti-SARS-CoV-2 Spike and RBD IgG were measured by a multiplex immunoassay on the MSD platform at PPD Laboratories. The assay sequences were based on the ancestral sequences from Wuhan, China. Antigen information and sequence information are provided in Supplementary Table 1. Assay validation included precision and ruggedness, dilutional linearity, selectivity, and relative accuracy for each SARS-CoV-2 antigens. Post-validation studies for stability and for conversion to the WHO standard, as well as the establishment of a cut-point, were performed. The lower limit of quantifications (LLOQs) for anti-spike and anti-RBD are 33 and $204 \mathrm{AU} / \mathrm{ml}$, respectively.

Antibody neutralization was measured with a lentivirus-based pseudovirus particle expressing the D614 SARS-CoV-2 spike protein. The pseudovirus neutralizing antibody assay was validated at Monogram Biosciences. Validation included accuracy, repeatability, intermediate precision, linearity, specificity/ selectivity, sensitivity, and stability utilizing pooled sera from high-titer, intermediate-titer, and low-titer pooled convalescent SARS-CoV-2 sera, as well as historical negative samples collected in the year 2017 (prior to SARS-CoV-2 circulation). The LLOQ for pseudovirus neutralizing antibody is $40\left(\mathrm{ID}_{50}\right)$.

Antibody neutralization was also measured by a live microneutralization assay using the Victoria/01/2020 strain of the virus (Public Health England). Qualification of the assay included assessment of specificity, parallelism, dilutional linearity, repeatability, intermediate precision, and assessment of the assay range. A formal validation has since been completed (after the testing of clinical study samples in this manuscript). Normalized values $\left(\mathrm{NF}_{50}\right)$ were used for the main analyses, as the normalization process removes the plate-to-plate variability and normalized values are more highly correlated with binding antibody and pseudovirus neutralization assays. However, normalized values cannot be converted into WHO standard units. A sensitivity analysis is provided in Supplementary Table 4 using non-normalized values $\left(\mathrm{ND}_{50}\right)$, which are also presented as IU/ml using the WHO standard, but are less highly correlated with other assays. The LLOQ of the assay is $58\left(\mathrm{ND}_{50}\right)$ and $8.6\left(\mathrm{NF}_{50}\right)$.

Due to the limitations of laboratory capacity, fewer samples were tested for virus neutralization than were tested using the quicker multiplex assay.
Imputation on censored immune marker data in main analysis. Immune marker values were $\log _{10}$-transformed prior to analysis. Values that were censored at the lower limit of quantification (LLOQ) were imputed with the value LLOQ/2. Approximately $10 \%$ of the pseudovirus neutralization titer was censored at the LLOQ, and sensitivity analyses were conducted by imputing these values using a Gibbs sampler.

Conversion to WHO International Standard (20/136). Each assay was analyzed in its original scale, and results were then converted to the WHO international standard units using the conversion factors supplied by each laboratory. WHO standard units are BAU/ml for anti-spike and anti-RBD IgG, and IU $/ \mathrm{ml}$ for neutralization titers ${ }^{40}$. For PPD conversion, factors are supplied with CIs. These are not able to be applied to the converted data, as it is a one-to-one conversion. For the Monogram assay, multiple forms of the conversion factor were supplied, and all three were implemented.

Conversion factors were as follows:

PPD: Conversion from $\mathrm{AU} / \mathrm{ml}$ to $\mathrm{BAU} / \mathrm{ml}$

- Anti-spike IgG 0.00645, 95\% CI $(0.00594,0.00701)$

- Anti-RBD IgG 0.00798, 95\% CI $(0.00735,0.00866)$

Monogram pseudovirus neutralization assay (D614) conversion from $\mathrm{ND}_{50}$ to $\mathrm{IU} / \mathrm{ml}$

- 0.1428 (mean)

- 0.1458 (geometric mean)

- 0.1534 (median)

PHE live-virus neutralization assay conversion from $\mathrm{ID}_{50}$ to $\mathrm{IU} / \mathrm{ml}$

- $0.2461(1 / 4.064)$

Study design and analysis populations. We first defined the correlates population by restricting it to participants who met the eligibility criteria and received ChAdOx1 nCoV-19: participants were eligible for inclusion if they were baseline seronegative to the SARS-CoV-2 N protein at first vaccination, had their PB28 visit within a 14- to 42-day window after the second dose, and were followed up to at least 7 days after PB28, with no prior evidence of infection. Participants were excluded from analysis if infection occurred before PB28. Participants who received two doses were included in the analysis, either standard dose followed by standard dose (SD/SD), or low dose followed by low or standard dose (LD/SD or LD/LD). Nine participants who received mixed schedules (one dose of ChAdOx1 nCoV-19 and one dose of MenACWY control) in error were excluded from analysis (Extended Data Fig. 1). The same eligibility criteria were applied to define a control population of MenACWY recipients.

Among the ChAdOx1 nCoV-19 correlates population, those who had biomarker data available comprised the correlates cohort. Participants who tested NAAT + more than 7 days after PB28 were defined as cases, while those who did not have a positive test were defined as noncases. The 7-day window was implemented to exclude cases in which exposure is likely to have occurred before a blood sample was taken.

Statistical Analysis. Baseline exposure risk to SARS-CoV-2 infections. To control for potential confounding due to variation in exposure risk among participants with available immune marker data, a logistic regression risk model was developed among the control population of MenACWY recipients. Baseline factors associated with exposure risk were used to model the probability of being NAAT+ in this population. Baseline variables for the risk model included age in years, ethnicity (white and nonwhite), BMI $\left(<30 \mathrm{~kg} / \mathrm{m}^{2}, \geq 30 \mathrm{~kg} / \mathrm{m}^{2}\right)$, comorbidities (having any of: respiratory disease; cardiovascular disease; or diabetes), and healthcare worker status (nonhealthcare worker, healthcare worker exposed to no more than one patient with COVID-19 on an average day; healthcare worker exposed to one or more patients with COVID-19 on an average day). Output is shown in Supplementary Table 3. The linear predictor from the risk model developed using the MenACWY control population was used to predict the baseline risk of exposure in the ChAdOx1 nCoV-19 correlates cohort.

Correlates or risk. The correlates of risk (CoR) analysis was conducted within the correlates cohort. log-transformed immune marker values were analyzed using generalized additive models (GAM) for binary data, with a cubic spline smooth applied to immune marker values to allow a nonlinear effect. The logit-transformed predicted baseline exposure risk was included as a linear covariate in the GAM model. A $P$ value $<0.05$ from the approximate significance test from the smooth GAM was used to determine if an immune marker was associated with protection. There was no adjustment for multiple comparison. Separate models were fitted for each immune marker controlling for baseline exposure risk, and weighted by inverse probability weights as described below.

Inverse probability weighting. Immune marker data were not available for everyone in the correlates population, and cases are over-represented in the immune marker datasets as these were preferentially processed over noncases. Unadjusted estimates of absolute risk of infection will therefore be inflated and result in bias to correlates estimates. We used a logistic regression model to predict the probability that a 
participant will have immune marker data available to the analysis. The outcome variables were each immune marker, and predictors were age group (18-55 years, 56-69 years, 70 years or above), whether the participant is a case or noncase, the type of case (primary symptomatic, nonprimary symptomatic, asymptomatic), prime-boost interval, and dosage (LD/LD, LD/SD, SD/SD). The inverse probability from this model was used to weight the correlates of risk models for each immune marker to remove this source of bias (Supplementary Table 3).

Correlates of vaccine efficacy. For each outcome, to derive the relative risk (RR) and correlates of vaccine efficacy, an estimate of the absolute averaged predicted risk from the CoR model was computed. The averaged absolute risk was then compared to the overall risk among MenACWY Correlates Population, which was itself weighted by the randomization ratio for study groups not randomized 1:1.

VE was defined as $100 \% \times(1-R R)$. Mean estimate of VE at each level of antibody in the dataset, as well as $95 \%$ CIs were calculated from 10,000 bootstrap samples.

Further analysis details are provided with the original trial statistical analysis plan (SAP) and the separate SAP developed for immune correlates analyses. The immune correlates SAP leant heavily on the methods proposed in the publicly available SAP by the Coronavirus Prevention Network (CoVPN) Biostatistics Team ${ }^{26}$.

Bootstrap. We resampled from all participants enrolled in the study. For each bootstrap sample, we calculated the inverse probability weights to account for sampling bias. We then estimated the CoR by GAM, adjusting for the baseline risk exposure and weighting by inverse probability weights. We compared the predicted absolute risk from the GAM across the full range of antibody values, with the resampled MenACWY control population weighted overall risk. Ten thousand bootstrap samples were used for each immune marker and outcome. The overall estimates for CoR and correlates of vaccine efficacy were given by the median value in the bootstrap; 95\% CIs were calculated using the bootstrap percentile method, that is, the $2.5 \%$ and $97.5 \%$ quantiles from the bootstrap.

Correlates and their CIs were not computed for assays in which the relationship between antibody and outcome was nonsignificant. Where CIs were outside the range of values of the assay, these are reported as NC.

Sensitivity analyses. Viral load. To account for potential of misclassification in asymptomatic infections, a sensitivity analysis was conducted excluding cases with lower viral loads (defined as those for whom all returned PCR positive tests had a CT value $\geq 30$ ), as these are potential false positives.

Imputation of censored antibody values. Approximately $10 \%$ of the pseudovirus neutralization antibody titers were below the LLOQ. We performed a sensitivity analysis to account for the potential bias caused by imputing LLOQ / 2. Studies have shown that imputing LLOQ / 2 can lead to bias and CIs with poor coverage when a substantial proportion of the data are censored ${ }^{41-43}$. When a bootstrap is required for missing data, Brand et al. found single imputation embedded inside a bootstrap showed better statistical properties than other methods ${ }^{43}$. We used an iterative Gibbs sampler proposed by Chen et al. to impute the censored log pseudovirus neutralization antibody values ${ }^{42}$.

Not all participants with results from the pseudovirus neutralization titer also have results from the anti-spike, anti-RBD, and live neutralizing antibody titers. For each bootstrap sample, we iteratively predicted the missing and censored values for each antibody titers in a Gibbs sampler, constraining the predictions for the censored values to be less than or equal to the LLOQ. The antibody titers were iteratively predicted by a sequence of Bayesian linear regressions. For each regression, the independent variables were the current prediction for all other titers, the baseline risk score and all variables used in the inverse probability weighting model.

Let $Z_{j}, j=1,2,3,4$ be the vector of the $j$ th antibody titers values. Let $\sigma_{j}^{2}$ and $\boldsymbol{\beta}_{j}$ be the variance and vector of regression parameters for the $j$ th linear regression update, respectively. We chose a noninformative prior $^{42,44}$ for $\sigma_{j}^{2}$ and $\boldsymbol{\beta}_{j}$, namely

$$
p\left(\sigma_{j}^{2}, \boldsymbol{\beta}_{j}\right) \propto \frac{1}{\sigma_{j}^{2}}
$$

Then the Gibbs sampler proposed by Chen et al. is as follows: ${ }^{42}$

Initialize the missing and censored values $Z_{j}^{(0)}$ for each $Z_{j}, j=1,2,3,4$.

For $i=1, \ldots, N$

For $j=1,2,3,4$

Update $\sigma_{j}^{2}$ and $\boldsymbol{\beta}_{j}$ from the posterior distribution given the current predictors for all other antibody values $Z_{\mathrm{k}}^{(i)}, k<j ; Z_{\mathrm{k}}^{(i-1)}, \mathrm{k}>j$ and the fixed covariates.

Update $Z_{j}^{(i)}$ from the posterior predictive distribution given $\sigma_{j}^{2}$ and $\boldsymbol{\beta}_{j}$ and the current values of the predictor variables.

We imputed a single value for each of the censored log pseudovirus neutralization antibody values from the $n=100$ th iteration of the Gibbs sampler. Note participants with missing log pseudovirus neutralization antibody titer values were excluded from the sensitivity analysis. The sensitivity analysis was then run on the imputed dataset for the bootstrap sample.
We initialized the Gibbs sampler by predicting the missing and censored values from a sequence of linear regressions on the nonmissing data. This sequence was developed with the data structure in mind, aiming to initialize the chain as close to the posterior mode as possible.

We ran multiple chains on bootstrap samples and tested for convergence by inspecting trace plots of the censored log pseudovirus neutralization titers. From these plots, we determined the 100th iteration to be approximately converged.

Data cut-off. The data cut-off date for inclusion in this analysis was 28 February 2021. Cases occurring after this date are not included in the analysis.

Software. Data analysis was done using R version 3.6.1 (ref. ${ }^{45}$ ). The GAM was coded using the mgcv package ${ }^{46}$. Three knots were used for each GAM, and the smoothing parameter was estimated by generalized crossvalidation.

Reporting Summary. Further information on research design is available in the Nature Research Reporting Summary linked to this article.

\section{Data availability}

Anonymized participant data will be made available when the trials are complete, upon requests directed to the corresponding author. Proposals will be reviewed and approved by the sponsor, investigator, and collaborators on the basis of scientific merit. After approval of a proposal, data can be shared through a secure online platform after signing a data access agreement. All data will be made available for a minimum of 5 years from the end of the trial.

\section{Code availability}

The $\mathrm{R}$ code for the main correlates estimates is available in Supplementary File (item 5, page 8).

\section{References}

38. Ramasamy, M. N. et al. Safety and immunogenicity of ChAdOxl nCoV-19 vaccine administered in a prime-boost regimen in young and old adults (COV002): a single-blind, randomised, controlled, phase 2/3 trial. Lancet 396 1979-1993 (2020)

39. Sterne, J. A. C. et al. Multiple imputation for missing data in epidemiological and clinical research: potential and pitfalls. Brit. Med. J. 338, b2393 (2009).

40. World Health Organization - National Institute for Biological Standards and Control. WHO International Standard: First WHO International Standard for anti-SARS-CoV-2 immunoglobulin (human) - NIBSC code: 20/136: Instructions for Use, v2.0. (2020).

41. Lubin, J. H. et al. Epidemiologic evaluation of measurement data in the presence of detection limits. Environ. Health Perspect. 112, 1691-1696 (2004).

42. Chen, H., Quandt, S. A., Grzywacz, J. G. \& Arcury, T. A. A Bayesian multiple imputation method for handling longitudinal pesticide data with values below the limit of detection. Environmetrics 24, 132-142 (2013).

43. Brand, J., van Buuren, S., le Cessie, S. \& van den Hout, W. Combining multiple imputation and bootstrap in the analysis of cost-effectiveness trial data. Stat. Med. 38, 210-220 (2019).

44. Box, G. E. \& Tiao, G. C. Bayesian Inference in Statistical Analysis (John Wiley \& Sons, 2011).

45. R Core Team. R: A language and environment for statistical computing. in $R$ Foundation for Statistical Computing. (2020).

46. Wood, S. N. Fast stable restricted maximum likelihood and marginal likelihood estimation of semiparametric generalized linear models. J. R. Stat. Soc. B 73, 3-36 (2011).

\section{Acknowledgements}

This article reports independent research funded by UK Research and Innovation (MC_PC_19055: SG, AJP, TL), Engineering and Physical Sciences Research Council (EP/R013756/1: S.C.G., A.J.P., T.L.), National Institute for Health Research (COV19 OxfordVacc-01: S.C.G., A.J.P., T.L.), Coalition for Epidemic Preparedness Innovations (Outbreak Response To Novel Coronavirus (COVID-19: S.C.G., A.J.P., T.L.)), National Institute for Health Research Oxford Biomedical Research Centre (BRC4 Vaccines Theme), Thames Valley and South Midland's NIHR Clinical Research Network, and AstraZeneca. The views expressed in this publication are those of the authors and not necessarily those of the NIHR or the UK Department of Health and Social Care. We acknowledge support from Thames Valley and South Midland's NIHR Clinical Research Network and the staff and resources of NIHR Southampton Clinical Research Facility and the NIHR Oxford Biomedical Research Centre. A.J.P. is a NIHR Senior Investigator The views expressed in this publication are those of the authors and not necessarily those of the NIHR or the UK Department of Health and Social Care. AstraZeneca reviewed the final manuscript but the academic authors retained editorial control. Other funders had no role in study design, data collection and analysis, or decision to publish. We thank the volunteers who participated in this study. We thank P. B. Gilbert and P. Dull for their advice and contributions to the methodology. The authors appreciate the efforts of the Labcorp-Monogram Biosciences Clinical Reference Laboratory. 


\section{Author contributions}

M.V. and S.F. designed the study. S.F., D.J.P., T.W., H.S., B.J., K.S., M.V., and I.H. contributed to the data analysis and methods. P.K.A., S.B., C.D., S.C.G., M.F., H.E.H. E.J.K., E.P., K.M.T., T.L.V., and J.V. contributed to implementation of the study and/or laboratory experimentation. S.F., M.V., T.L., and A.J.P. contributed to the preparation of the report. All authors critically reviewed and approved the final version.

\section{Competing interests}

Oxford University has entered into a partnership with AstraZeneca for further development of ChAdOx1 nCoV-19. S.C.G. is a cofounder of Vaccitech (collaborators in the early development of this vaccine candidate) and named as an inventor on a patent covering use of ChAdOx1 vectored vaccines and a patent application covering this SARS-CoV-2 vaccine (PCT/GB2012/000467). T.L. is named as an inventor on a patent application covering this SARS-CoV-2 vaccine and was a consultant to Vaccitech for an unrelated project during the conduct of the study. A.J.P. is chair of the UK Department of Health and Social Care (DHSC) Joint Committee on Vaccination \& Immunisation
(JCVI) but does not participate in discussions on COVID-19 vaccines, and is a member of WHO SAGE. The views expressed in this article do not necessarily represent the views of the DHSC, JCVI, NIHR, or WHO. T.W., H.S., I.H., J.B., E.J.K., K.S., J.V., and T.L.V. are employees of AstraZeneca. The other authors declare no competing interests.

\section{Additional information}

Extended data is available for this paper at https://doi.org/10.1038/s41591-021-01540-1. Supplementary information The online version contains supplementary material available at https://doi.org/10.1038/s41591-021-01540-1.

Correspondence and requests for materials should be addressed to Merryn Voysey.

Peer review information Nature Medicine thanks the anonymous reviewers for their contribution to the peer review of this work. Alison Farrell is the primary editor on this article and managed its editorial process and peer review in collaboration with the rest of the editorial team.

Reprints and permissions information is available at www.nature.com/reprints. 
Single blind immunogenicity and efficacy cohorts receiving ChAdOx1 n-CoV 19

\begin{tabular}{|c|}
\hline Group 1 \\
Age $56-69$ years \\
LD N=0 \\
LDLD N $=30$ \\
LDSD N $=30$ \\
\end{tabular}

\begin{tabular}{|l|}
\hline \multicolumn{1}{|c|}{ Excluded } \\
$\mathrm{N}=0$ baseline \\
serostatus \\
positive/NA \\
$\mathrm{N}=0$ single dose \\
recipients \\
$\mathrm{N}=0$ vaccine error \\
$\mathrm{N}=0$ cases $\leq 7$ days \\
since $\mathrm{PB28}$ \\
$\mathrm{N}=0$ follow-up $\leq 7$ \\
days since $\mathrm{PB} 28$ \\
$\mathrm{~N}=1$ samples not \\
collected or out of \\
$\mathrm{PB28}$ window \\
\end{tabular}

\begin{tabular}{|l|}
\hline \multicolumn{1}{|c|}{ Excluded } \\
$\mathrm{N}=1$ baseline \\
serostatus \\
positive/NA \\
$\mathrm{N}=8$ single dose \\
recipients \\
$\mathrm{N}=0$ vaccine error \\
$\mathrm{N}=0$ cases $\leq 7$ days \\
since $\mathrm{PB} 28$ \\
$\mathrm{~N}=0$ follow-up $\leq 7$ \\
days since $\mathrm{PB2} 28$ \\
$\mathrm{~N}=3$ samples not \\
collected or out of \\
$\mathrm{PB} 28$ window \\
\end{tabular}

\begin{tabular}{|c|}
\hline Eligible \\
$\mathrm{N}=59$ \\
Samples \\
processed \\
$\mathrm{N}=1$ cases \\
$\mathrm{N}=27$ non cases \\
\hline
\end{tabular}

Group 2
Age $70+$ years
LD N $=8$
LDLD N=46
LDSD N=46

\begin{tabular}{|c|}
\hline Group 4 \\
Age $18-55$ years \\
LD N $=242$ \\
LDLD N=51 \\
LDSD N $=1,424$ \\
\hline
\end{tabular}

Group 5
Age $18-55$ years
LD N=2
SD N=46
LDSD N=63
SDSD N=64

\begin{tabular}{|c|}
\hline Group 6 \\
Age $18-55$ years \\
SD N=325 \\
SDSD N=1,973 \\
\end{tabular}

Group 7
Age $56-69$ years
SD N=30
SDSD N=30

\begin{tabular}{|c|}
\hline Group 8 \\
Age $70+$ years \\
SD N=51 \\
SDSD N=49 \\
\end{tabular}

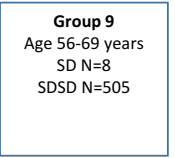

\begin{tabular}{|l|}
\hline \multicolumn{1}{|c|}{ Excluded } \\
$\mathrm{N}=0$ baseline \\
serostatus \\
positive/ $\mathrm{NA}$ \\
$\mathrm{N}=30$ single dose \\
recipients \\
$\mathrm{N}=0$ vaccine error \\
$\mathrm{N}=0$ cases $\leq 7$ days \\
since $\mathrm{PB28}$ \\
$\mathrm{N}=0$ follow-up $\leq 7$ \\
days since $\mathrm{PB} 28$ \\
$\mathrm{~N}=2$ samples not \\
collected or out of \\
$\mathrm{PB28}$ window
\end{tabular}

\begin{tabular}{|l|}
\hline \multicolumn{1}{|c|}{ Excluded } \\
$\mathrm{N}=2$ baseline \\
serostatus \\
positive/NA \\
$\mathrm{N}=50$ single dose \\
recipients \\
$\mathrm{N}=0$ vaccine error \\
$\mathrm{N}=0$ cases $\leq 7$ days \\
since $\mathrm{PB2} 28$ \\
$\mathrm{~N}=0$ follow-up $\leq 7$ \\
days since $\mathrm{PB} 28$ \\
$\mathrm{~N}=0$ samples not \\
collected or out of \\
$\mathrm{PB} 28$ window \\
\end{tabular}

\begin{tabular}{|l|}
\hline \multicolumn{1}{|c|}{ Excluded } \\
$\mathrm{N}=22$ baseline \\
serostatus \\
positive/NA \\
$\mathrm{N}=8$ single dose \\
recipients \\
$\mathrm{N}=2$ vaccine error \\
$\mathrm{N}=8$ cases $\leq 7$ days \\
since $\mathrm{PB28}$ \\
$\mathrm{N}=6$ follow-up $\leq 7$ \\
days since $\mathrm{PB} 28$ \\
$\mathrm{~N}=14$ samples not \\
collected or out of \\
$\mathrm{PB} 28$ window \\
\end{tabular}

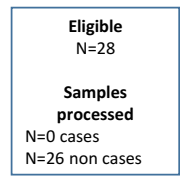

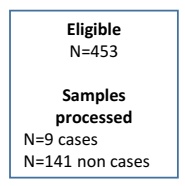

$\mathrm{N}=141$ non cases

\begin{tabular}{|l|}
\hline \multicolumn{1}{|c|}{ Excluded } \\
$\mathrm{N}=23$ baseline \\
serostatus \\
positive/NA \\
$\mathrm{N}=236$ single dose \\
recipients \\
$\mathrm{N}=2$ vaccine error \\
$\mathrm{N}=13$ cases $\leq 7$ \\
days since $\mathrm{PB} 28$ \\
$\mathrm{~N}=10$ follow-up $\leq 7$ \\
days since $\mathrm{PB} 28$ \\
$\mathrm{~N}=79$ samples not \\
collected or out of \\
$\mathrm{PB2} 2$ window \\
\end{tabular}

\section{$\mathrm{N}=32$ baseline}

serostatus

recipients

$\mathrm{N}=0$ vaccine error

$\mathrm{N}=2$ cases $\leq 7$ days

since PB28

$\mathrm{N}=2$ follow-up $\leq 7$
days since PB28

$\mathrm{N}=0$ samples not

collected or out of

out of PB28

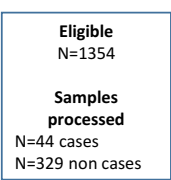

$\mathrm{N}=48$ single dose

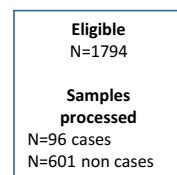

$\mathrm{N}=26$ non

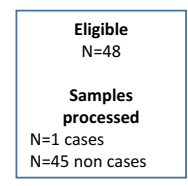

Group 10

Age $70+$ years
SD N $=5$

SDSD N=508

\begin{tabular}{|l|}
\hline \multicolumn{1}{|c|}{ Excluded } \\
$\mathrm{N}=29$ baseline \\
serostatus \\
positive/NA \\
$\mathrm{N}=5$ single dose \\
recipients \\
$\mathrm{N}=1$ vaccine error \\
$\mathrm{N}=12$ cases $\leq 7$ \\
days since $\mathrm{PB} 28$ \\
$\mathrm{~N}=5$ follow- $\mathrm{up} \leq 7$ \\
days since $\mathrm{PB} 28$ \\
$\mathrm{~N}=4$ samples not \\
collected or out of \\
$\mathrm{PB} 28$ window \\
\\
\\
\\
\multicolumn{1}{|c|}{ Eligible } \\
$\mathrm{N}=457$ \\
\multicolumn{1}{|c|}{ Samples } \\
processed \\
$\mathrm{N}=11$ cases \\
$\mathrm{N}=117$ non cases \\
\hline
\end{tabular}

Extended Data Fig. 1 | Participant flow chart showing inclusion in correlates models. Eligible participants comprised the Correlates Population and those with samples processed comprised the Correlates Cohort. 
है

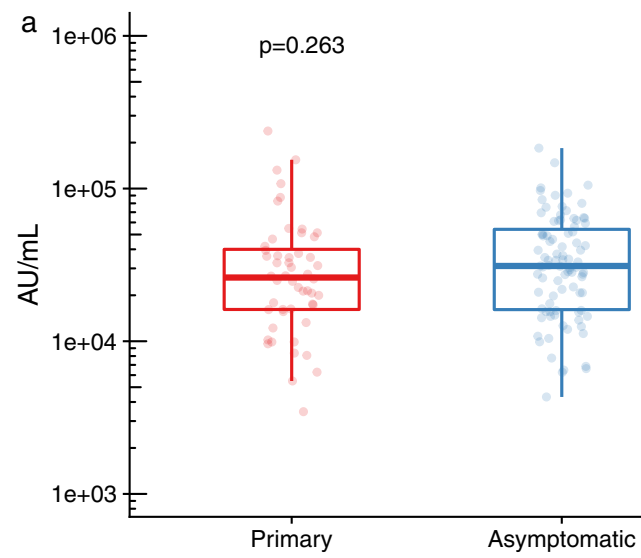

Anti-Spike IgG

$C_{1 e+06} \frac{1}{E} \quad p=0.507$

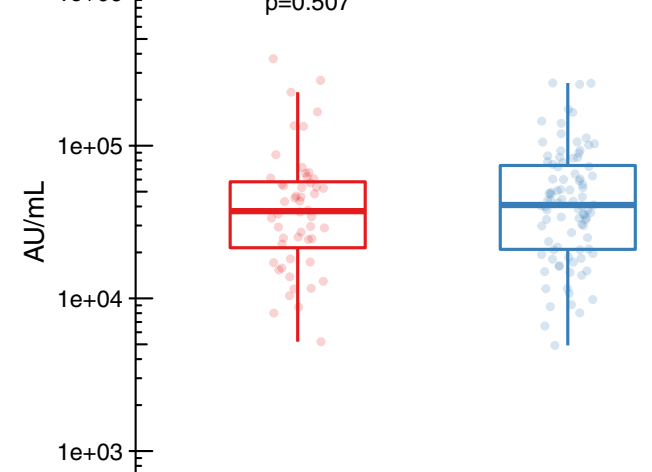

Primary

e $10000 \frac{\mathrm{t}}{\mathrm{E}} \quad \mathrm{p}=0.295$

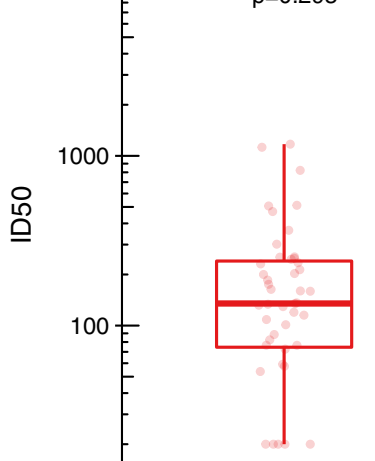

Primary

9

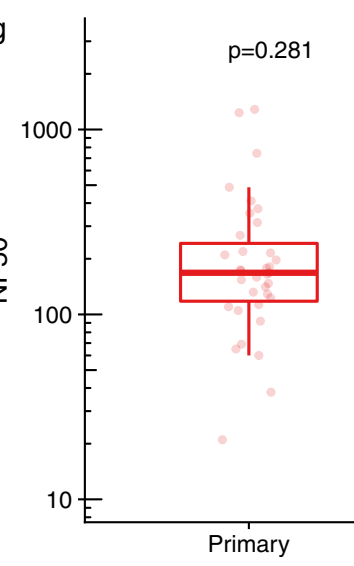

Asymptomatic

\section{Pseudoneutralisation titer}

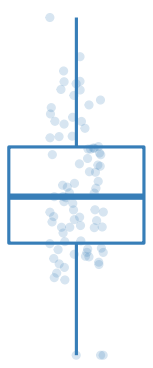

Asymptomatic

Live neutralisation titer

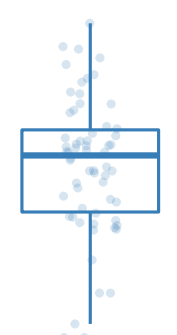

Asymptomatic

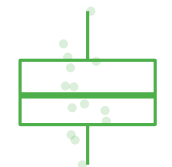

Anti-RBD IgG

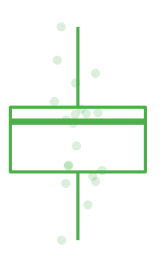

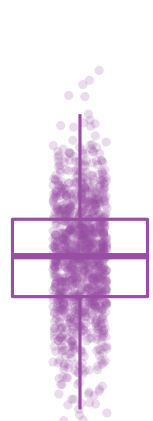

$1 e+03=$

NAAT negative

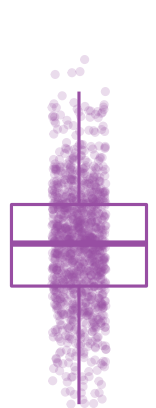

$\mathrm{d}_{1 \mathrm{e}+06}$

NAAT positive

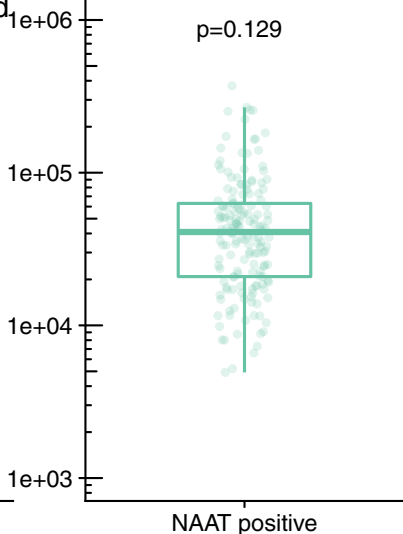

NAAT negative

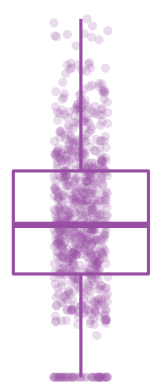

NAAT negative

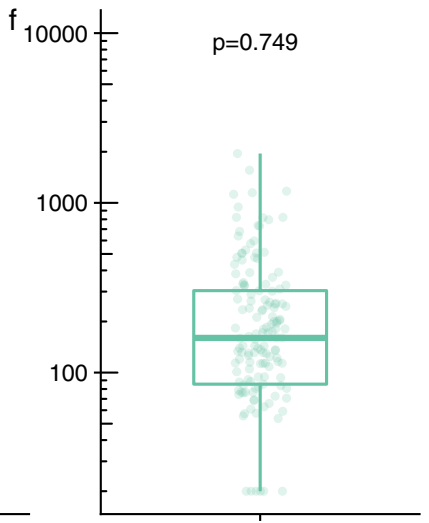

NAAT positive

h

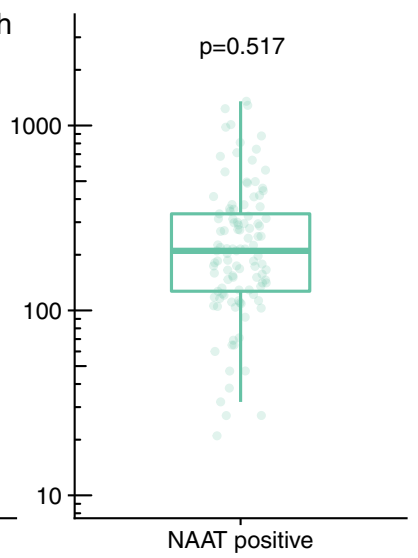

Extended Data Fig. 2 | See next page for caption. 


\section{NATURE MEDICINE}

Extended Data Fig. 2 | Immune markers measured at day 28 post-second dose, in primary symptomatic, asymptomatic, non-primary cases, NAAT positive cases and NAAT negative non-cases. a: $\mathrm{N}=1155$ NAAT negative, 52 primary, 91 asymptomatic and 20 non-primary, $\mathbf{b}$ : $\mathrm{N}=163 \mathrm{NAAT}$ positive participants' anti-spike lgG measured at 28 days post boost; $\mathbf{c}: \mathrm{N}=1155$ negative, 52 primary, 91 asymptomatic and 20 non-primary, d: $\mathbf{N}=163 \mathrm{NAAT}$ positive participants' anti-RBD IgG measured at 28 days post boost; $\mathbf{e}: \mathrm{N}=828$ NAAT negative, 47 primary, 86 asymptomatic and 16 non-primary, $\mathbf{f}: \mathrm{N}=$ 149 NAAT positive participants' pseudovirus neutralisation titre measured at 28 days post boost; $\mathbf{g}: \mathrm{N}=412$ negative, 36 primary, 62 asymptomatic and 12 non-primary, $\mathbf{h}: \mathrm{N}=110$ NAAT positive participants' live neutralisation titre measured at 28 days post boost. $\mathbf{a}-\mathbf{h}$ : minima: smallest value; maxima: largest value; centre: median value; bounds of box: $25 \%$ and $75 \%$ quartile value; upper/lower whisker extends from the hinge to the largest/smallest value no further than $1.5^{*}$ inter-quartile range from the hinge. IgG: Immunoglobulin G; RBD: receptor binding domain. Primary symptomatic cases: NAAT+ with at least one COVID symptom (cough, fever, shortness of breath, anosmia, aguesia). Asymptomatic cases: NAAT+ on weekly self-swab with no symptoms recorded. Non-primary cases: NAAT+ with only non-primary COVID symptoms (for example nausea, diarrhoea). P-value estimated by one-way ANOVA test comparing between primary, asymptomatic, non-primary cases and NAAT negative non-cases and by two sample t-test comparing between NAAT positive cases and NAAT negative non-cases (two-sided). 

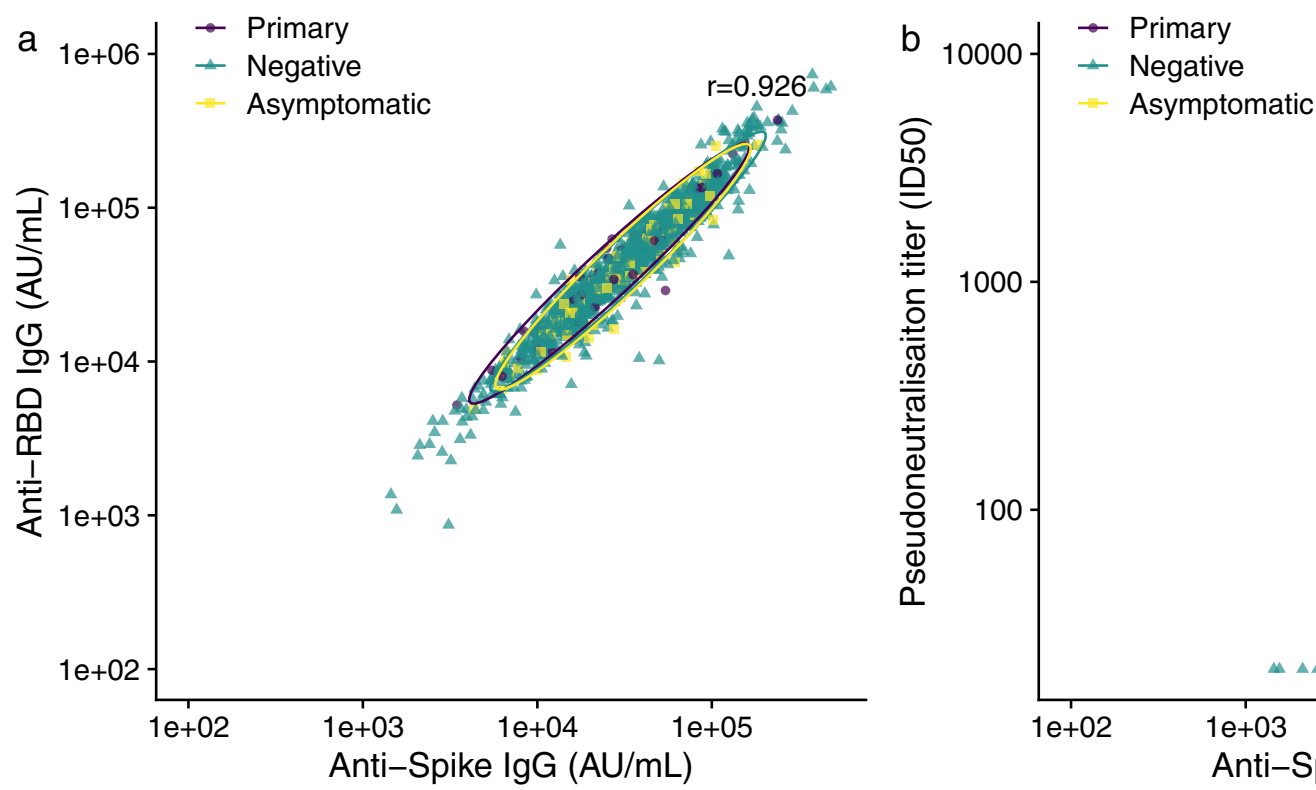

$r=0.657$

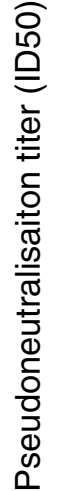

(1)
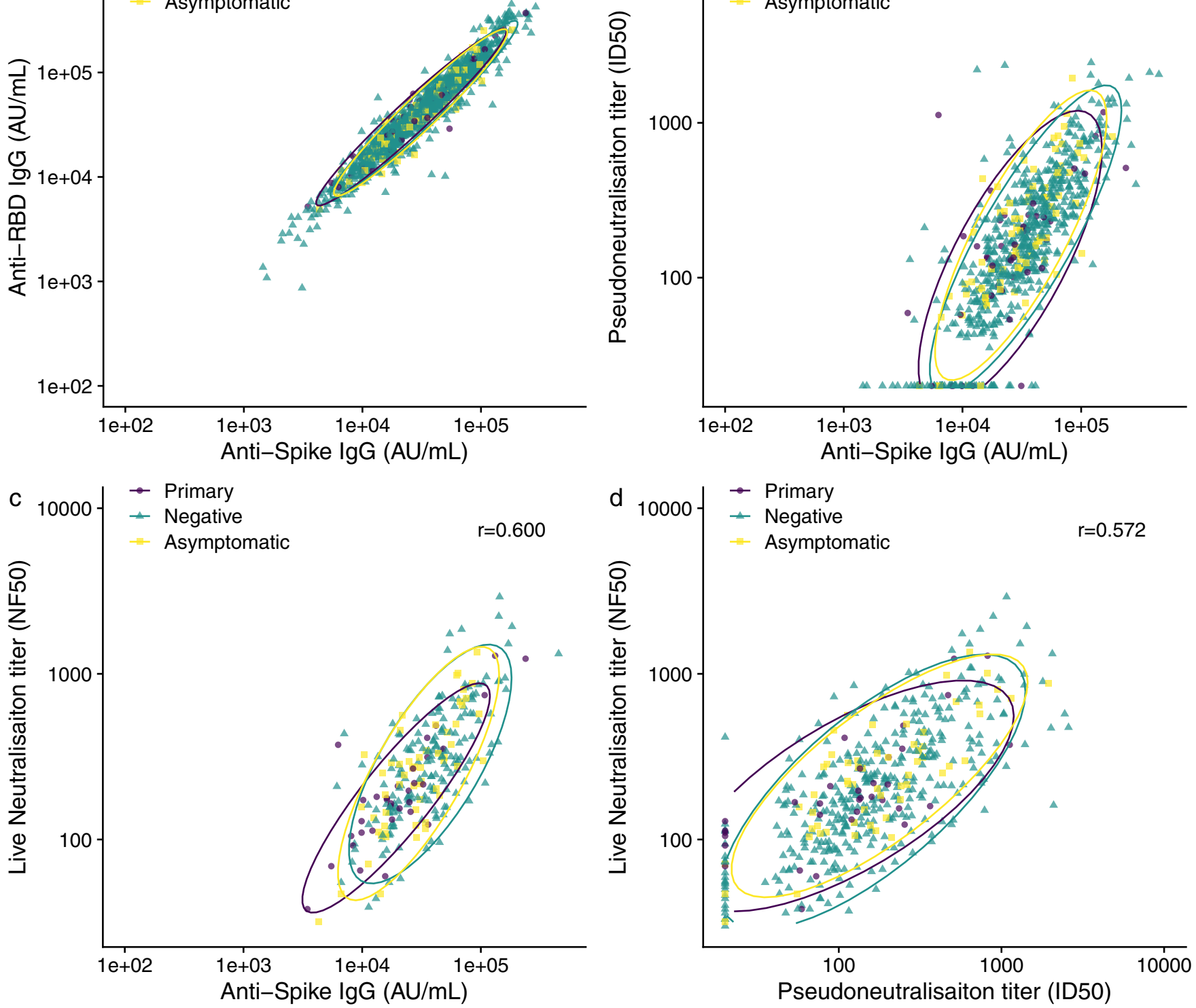

Extended Data Fig. 3 | Correlations between a, Anti-SARS-CoV-2 spike and RBD IgG. b, Anti-SARS-CoV-2 Spike IgG and pseudovirus neutralisation titre. c, Anti-SARS-CoV-2 Spike IgG and live virus neutralisation titre. d, pseudovirus neutralisation titres and live virus neutralisation titres. $95 \%$ confidence ellipses assuming a t-distribution are shown for each outcome (primary symptomatic cases, asymptomatic cases and negative controls). Pearson correlation coefficients shown as $r$ values using all available data. Primary symptomatic cases: NAAT+ with at least one COVID symptom (cough, fever, shortness of breath, anosmia, aguesia). Asymptomatic cases: NAAT+ on weekly self-swab with no symptoms recorded. 

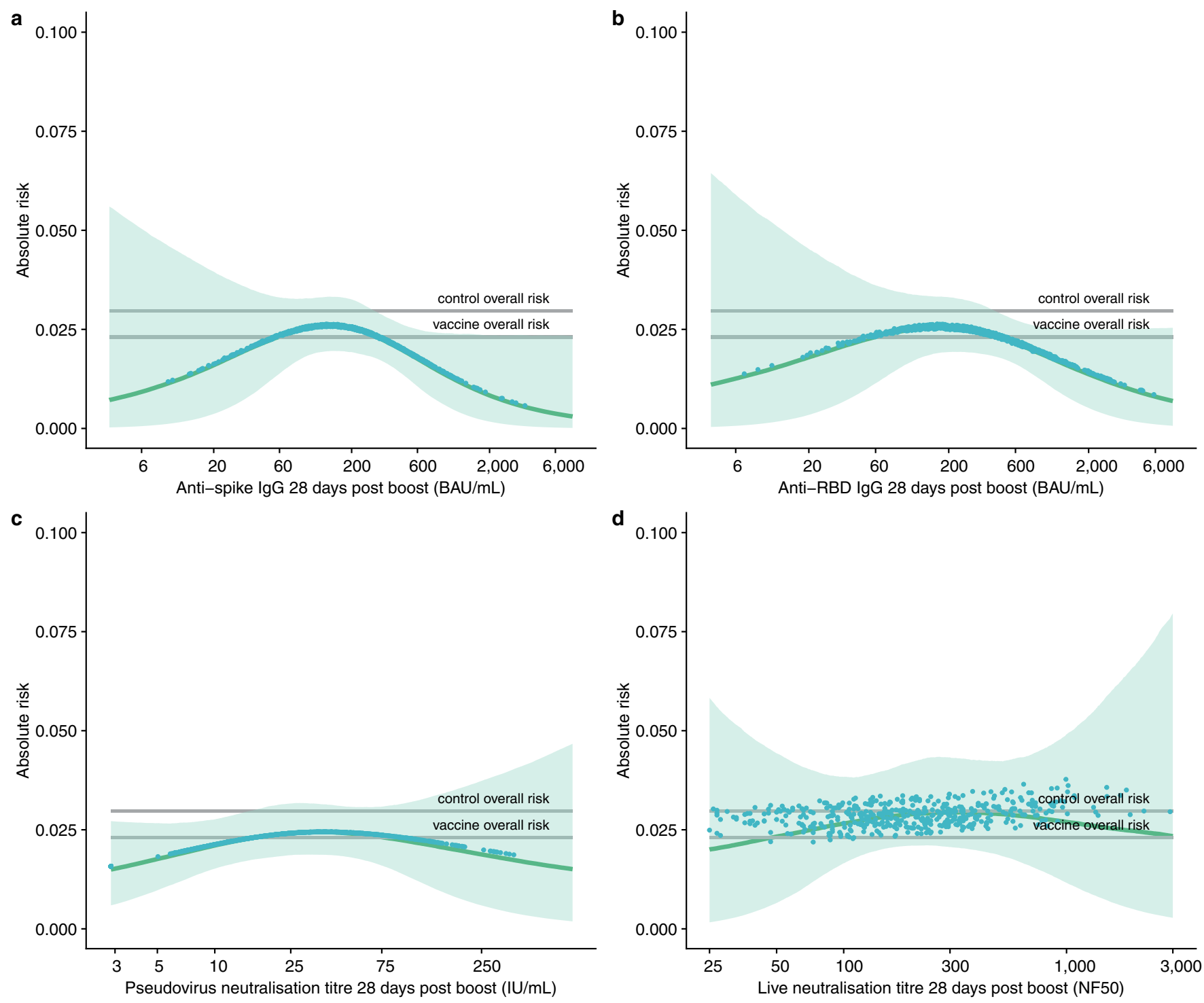

Extended Data Fig. 4 | Predicted absolute risk of asymptomatic SARS-CoV-2 infection as a function of immune markers measured 28 days post second dose. Predicted absolute risk of asymptomatic infection as a function of: a: Anti-spike lgG measured at 28 days post boost ( 91 cases, 1155 non-cases included in the analysis). b: Anti-RBD IgG measured at 28 days post boost ( 91 cases, 1155 non-cases included in the analysis). c: Pseudovirus neutralisation antibody titres 28 days post boost ( 86 cases, 828 non-cases included in the analysis). d: Live-virus neutralisation antibody titres 28 days post boost ( 62 cases, 412 non-cases included in the analysis). Grey horizontal lines show the overall risk of primary symptomatic COVID-19 in the control group (MenACWY) and vaccine groups (ChAdOx1 nCoV-19). Blue dots show the absolute risk predicted from the model across the range of antibody values included in the analysis, adjusting for baseline exposure risk to SARS-CoV-2 infection. Green shaded areas show the confidence interval around the predicted mean probability (green line). 

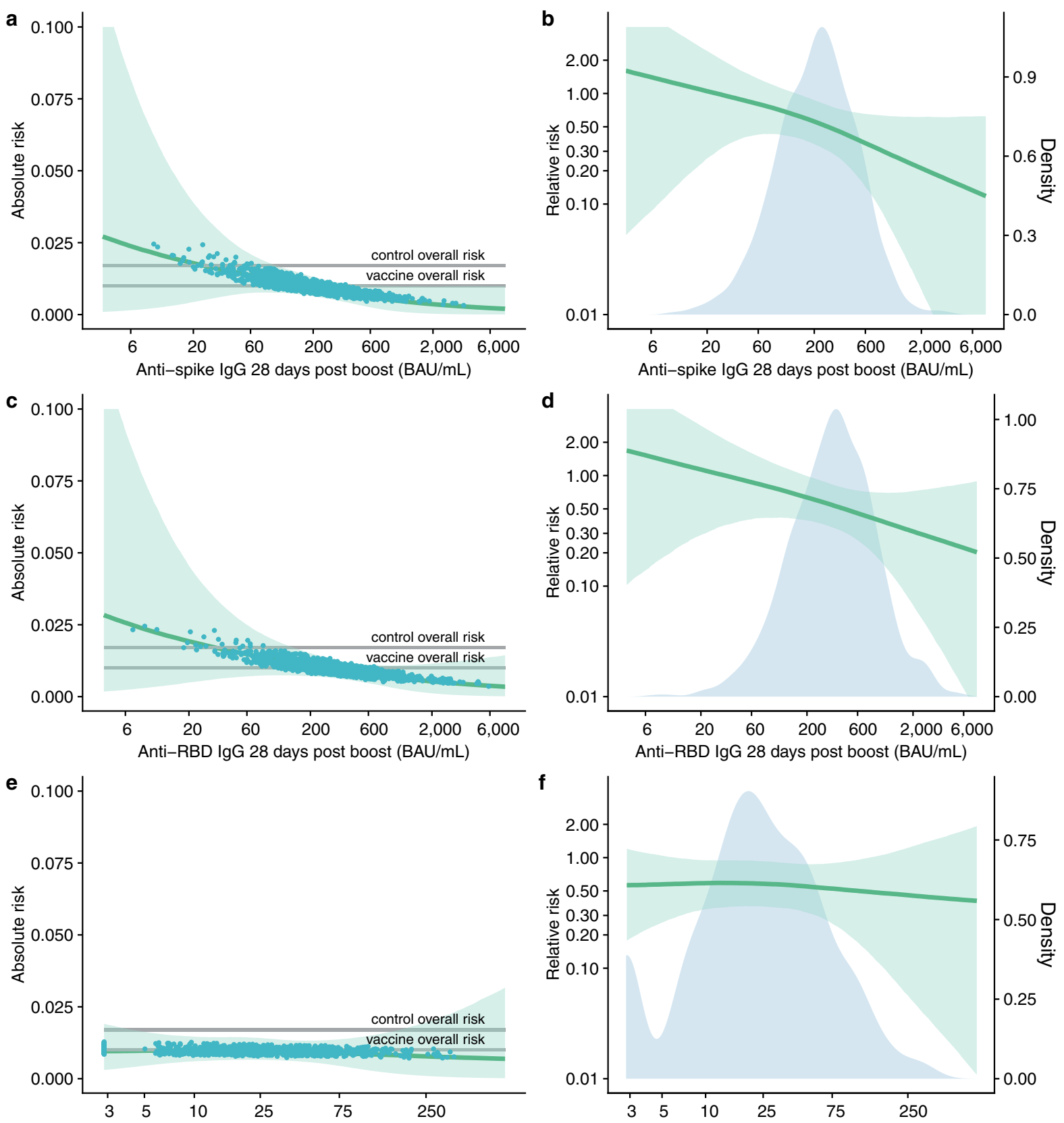

Pseudovirus neutralisation titre 28 days post boost $(\mathrm{IU} / \mathrm{mL})$

Pseudovirus neutralisation titre 28 days post boost (IU/mL)
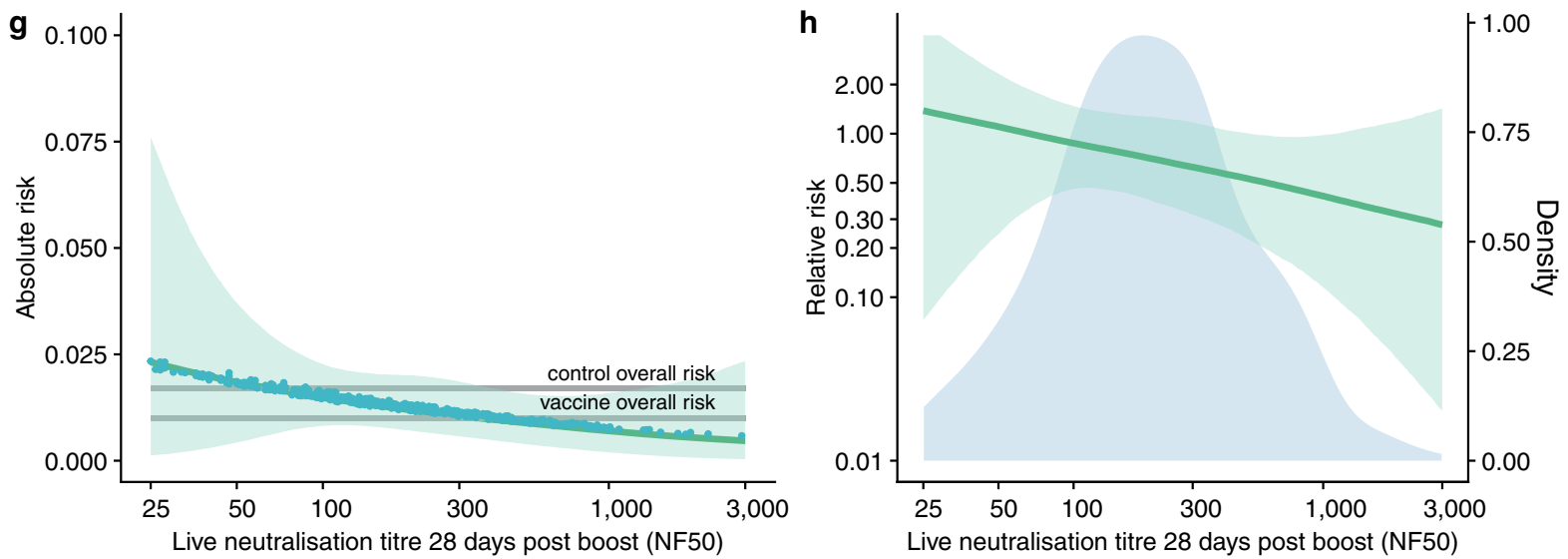

Extended Data Fig. 5 | See next page for caption. 
Extended Data Fig. 5 | Sensitivity analysis showing absolute and relative risk of asymptomatic SARS-CoV-2 infection as a function of immune markers measured at 28 days post second dose excluding cases with low viral load $(\mathbf{C t} \geq \mathbf{3 0})$. a, $\mathbf{c}, \mathbf{e}, \mathbf{g}$ : Grey horizontal lines show the overall risk of primary symptomatic COVID-19 in the control group (MenACWY) and vaccine groups (ChAdOx1 nCoV-19). Blue dots show the absolute risk predicted from the model across the range of antibody values included in the analysis, adjusting for baseline exposure risk to SARS-CoV- 2 infection. Green shaded areas show the confidence interval around the predicted mean probability (green line). $\mathbf{b}, \mathbf{d}, \mathbf{f}, \mathbf{h}$ : Blue shaded areas represent the immune marker density distribution. Green lines show the relative risk of infection among vaccine recipients compared to the MenACWY control arm participants. The green lines are the median relative risk obtained from 10,000 bootstrap samples. Green shaded areas are $95 \%$ bootstrapped confidence intervals for the relative risk. 

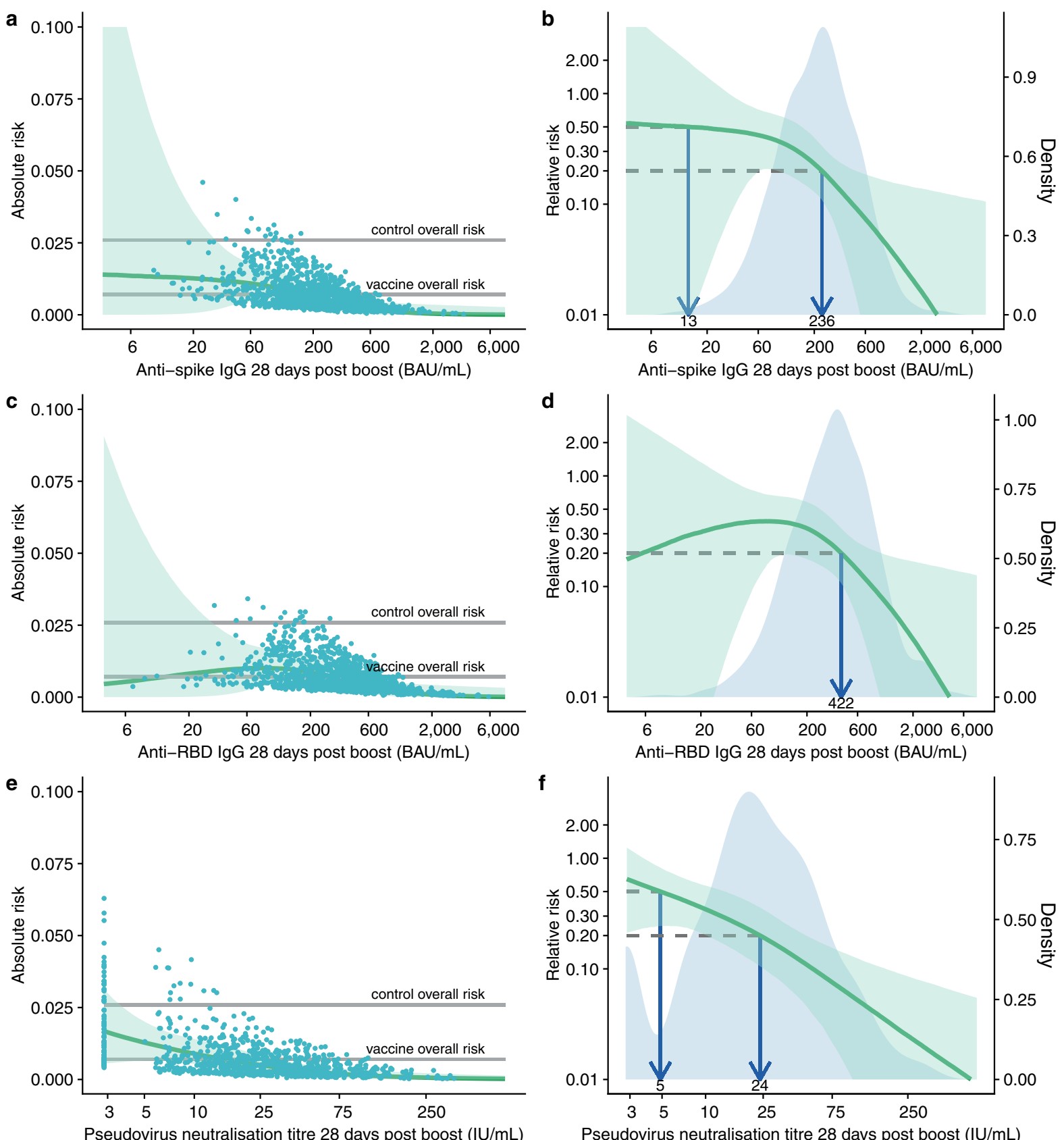

Pseudovirus neutralisation titre 28 days post boost (IU/mL)

Pseudovirus neutralisation titre 28 days post boost $(\mathrm{IU} / \mathrm{mL})$
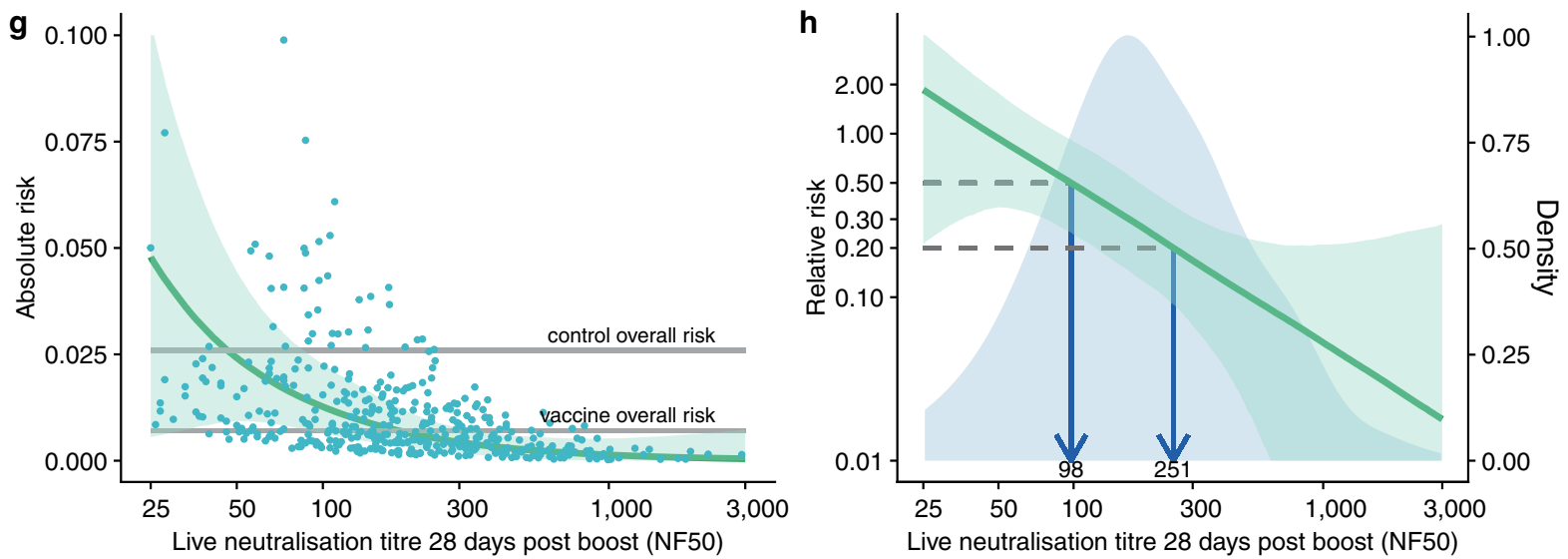

\section{Extended Data Fig. 6 | See next page for caption.}




\section{NATURE MEDICINE}

Extended Data Fig. 6 | Sensitivity analysis showing absolute and relative risk of primary symptomatic SARS-CoV-2 infection in participants with symptoms of shortness of breath as a function of immune markers measured at day $\mathbf{2 8}$ post-second dose. Results are shown for: $\mathbf{a}$, $\mathbf{b}$ : Anti-spike lgG measured at 28 days post boost ( 28 cases, 1155 non-cases included in the analysis). c, d: Anti-RBD lgG measured at 28 days post boost ( 28 cases, 1155 non-cases included in the analysis). e, f: Pseudovirus neutralisation antibody titres 28 days post boost (27 cases, 828 non-cases included in the analysis). $\mathbf{g}, \mathbf{h}$ : Live virus neutralisation antibody titres 28 days post boost (22 cases, 412 non-cases included in the analysis). a, c, e, g: Grey horizontal lines show the overall risk of primary symptomatic COVID-19 in the control group (MenACWY) and vaccine groups (ChAdOx1 nCoV-19). Blue dots show the absolute risk predicted from the model across the range of antibody values included in the analysis, adjusting for baseline exposure risk to SARS-CoV-2 infection. Green shaded areas show the confidence interval around the predicted mean probability (green line) b, $\mathbf{d}, \mathbf{f}, \mathbf{h}$ : Blue shaded areas represent the immune marker density distribution. Green lines show the relative risk of infection among vaccine recipients compared to the MenACWY control arm participants. The green lines are the median relative risk obtained from 10,000 bootstrap samples. Green shaded areas are $95 \%$ bootstrapped confidence intervals for the relative risk. 

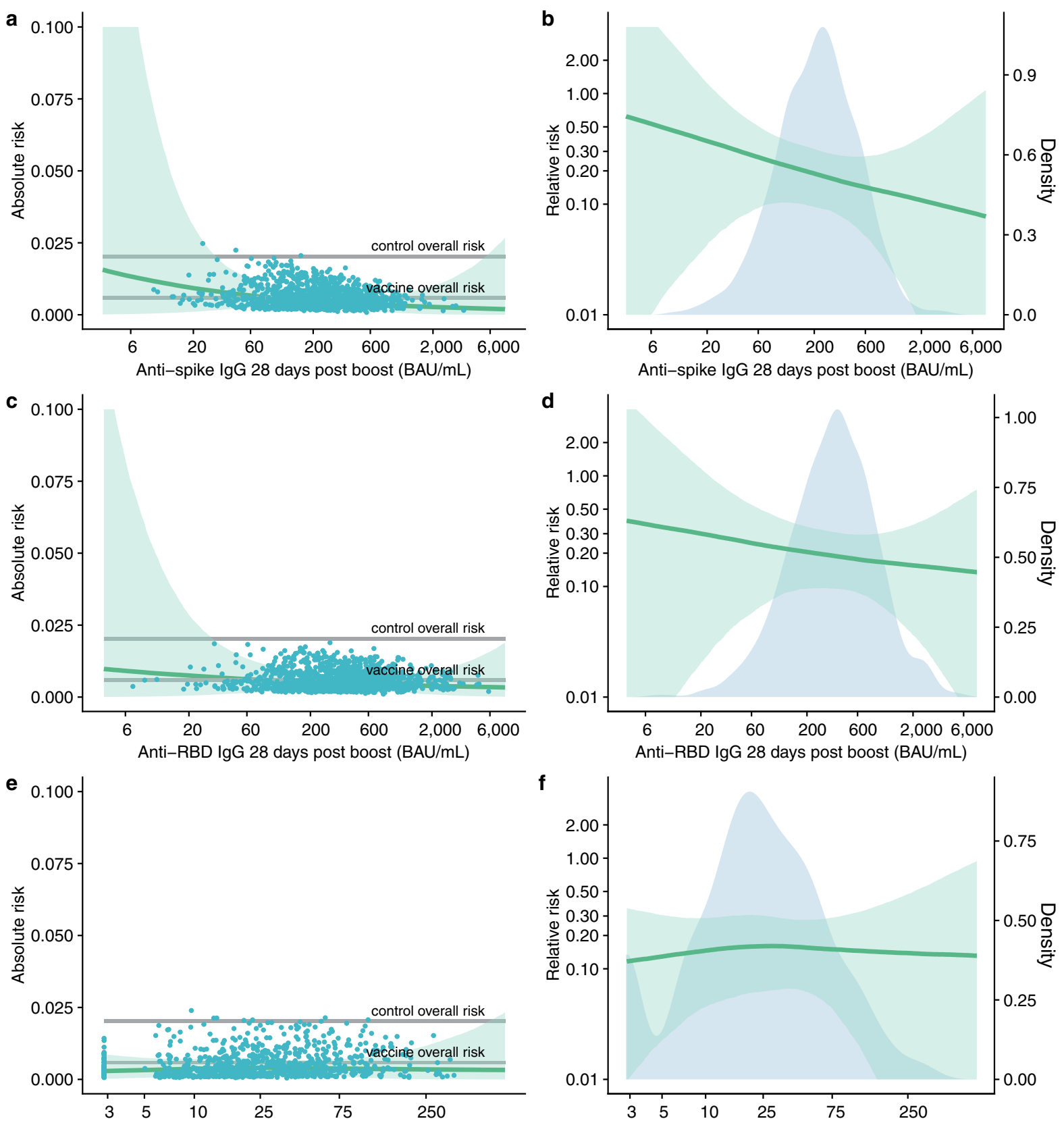

Pseudovirus neutralisation titre 28 days post boost $(\mathrm{IU} / \mathrm{mL})$

Pseudovirus neutralisation titre 28 days post boost (IU/mL)
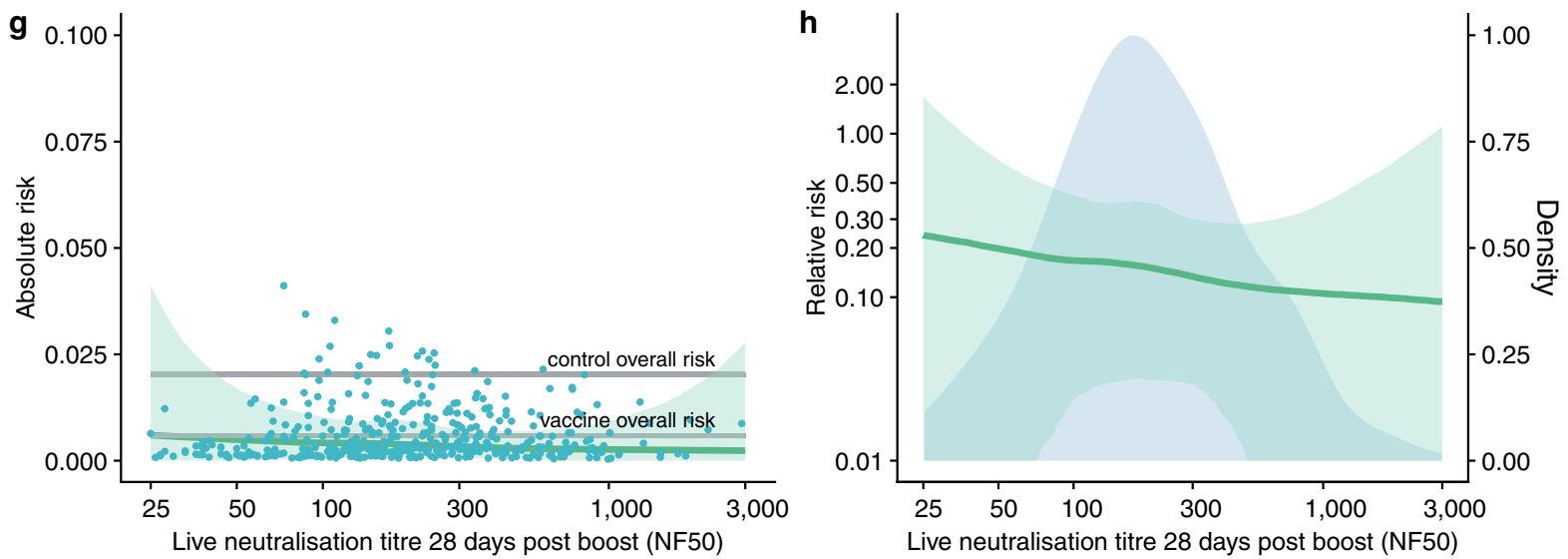

Extended Data Fig. 7 | See next page for caption. 


\section{NATURE MEDICINE}

Extended Data Fig. 7 | Sensitivity analysis showing absolute and relative risk of primary symptomatic SARS-CoV-2 infection in participants with no symptoms of shortness of breath as a function of immune markers measured at day $\mathbf{2 8}$ post-second dose. Results are shown for: $\mathbf{a}$, $\mathbf{b}$ : Anti-spike lgG measured at 28 days post boost ( 24 cases, 1155 non-cases included in the analysis). c, d: Anti-RBD IgG measured at 28 days post boost ( 24 cases, 1155 non-cases included in the analysis). e, f: Pseudovirus neutralisation antibody titres 28 days post boost (20 cases, 828 non-cases included in the analysis). $\mathbf{g}, \mathbf{h}$ : Live virus neutralisation antibody titres 28 days post boost (14 cases, 412 non-cases included in the analysis). a, c, e, $\mathbf{g}$ : Grey horizontal lines show the overall risk of primary symptomatic COVID-19 in the control group (MenACWY) and vaccine groups (ChAdOx1 nCoV-19). Blue dots show the absolute risk predicted from the model across the range of antibody values included in the analysis, adjusting for baseline exposure risk to SARS-CoV-2 infection. Green shaded areas show the confidence interval around the predicted mean probability (green line). $\mathbf{b}, \mathbf{d}, \mathbf{f}, \mathbf{h}$ : Blue shaded areas represent the immune marker density distribution. Green lines show the relative risk of infection among vaccine recipients compared to the MenACWY control arm participants. The green lines are the median relative risk obtained from 10,000 bootstrap samples. Green shaded areas are $95 \%$ bootstrapped confidence intervals for the relative risk. 

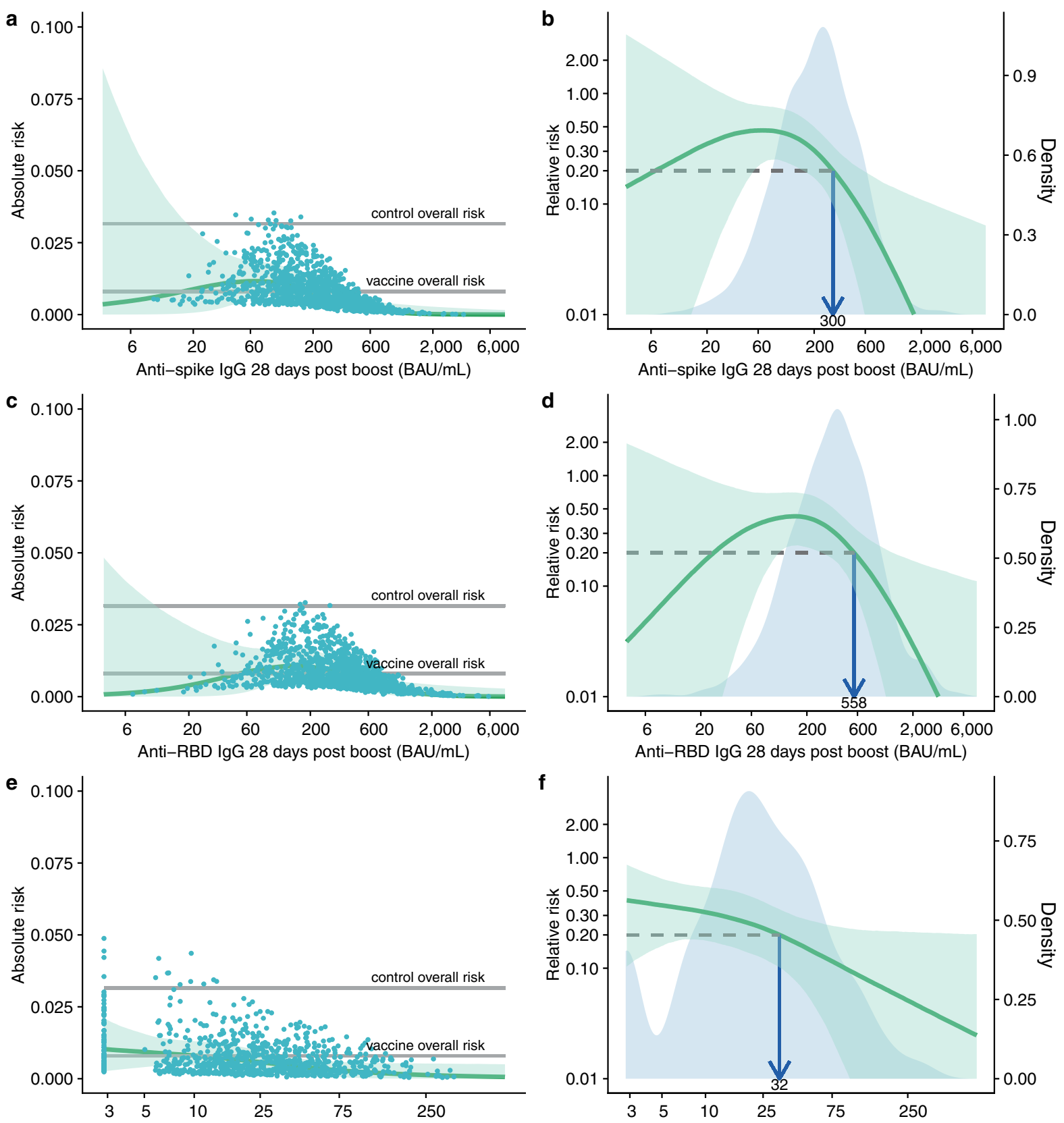

Pseudovirus neutralisation titre 28 days post boost $(\mathrm{IU} / \mathrm{mL})$

Pseudovirus neutralisation titre 28 days post boost (IU/mL)
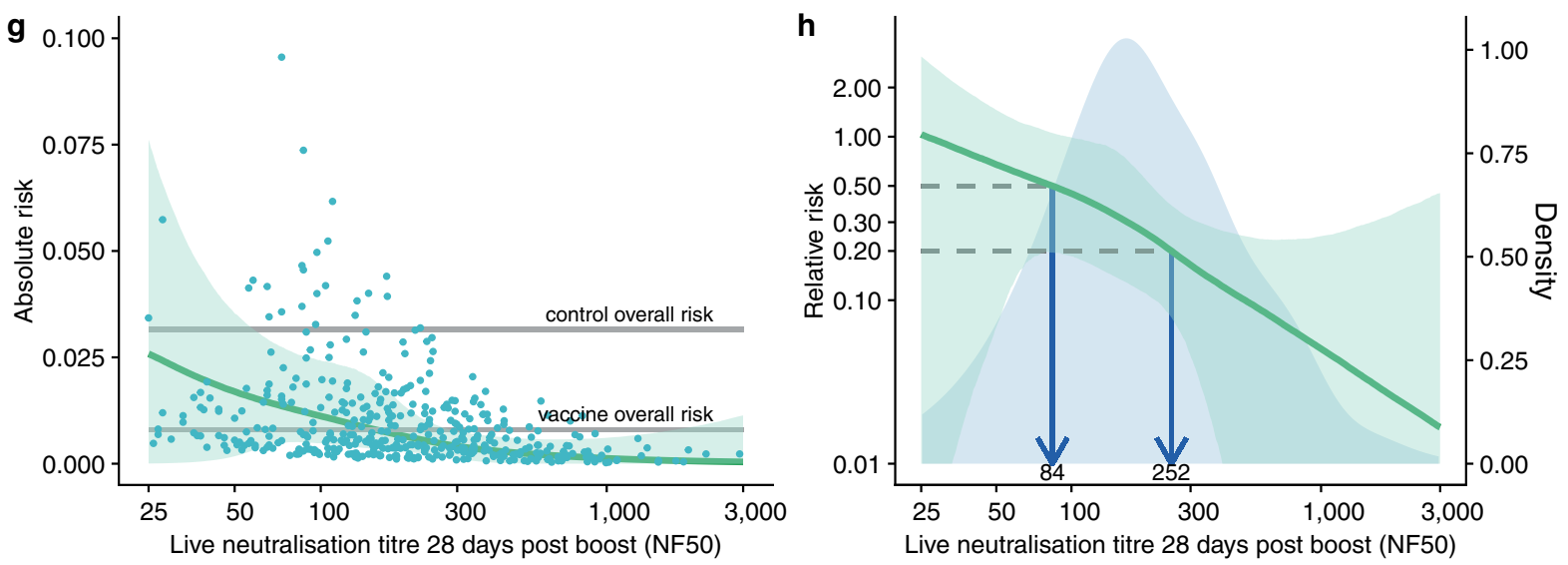

\section{Extended Data Fig. 8 | See next page for caption.}




\section{NATURE MEDICINE}

Extended Data Fig. 8 | Sensitivity analysis showing absolute and relative risk primary symptomatic SARS-CoV-2 infection with 3 or more COVID-19 symptoms as a function of immune markers measured at day 28 post-second dose. Results are shown for: $\mathbf{a}, \mathbf{b}$ : Anti-spike lgG measured at 28 days post boost (32 cases, 1155 non-cases included in the analysis). c, d: Anti-RBD lgG measured at 28 days post boost (32 cases, 1155 non-cases included in the analysis). e, f: Pseudovirus neutralisation antibody titres 28 days post boost ( 28 cases, 828 non-cases included in the analysis). $\mathbf{g}$, $\mathbf{h}$ : Live virus neutralisation antibody titres 28 days post boost (21 cases, 412 non-cases included in the analysis). a, c, e, g: Grey horizontal lines show the overall risk of primary symptomatic COVID-19 in the control group (MenACWY) and vaccine groups (ChAdOx1 nCoV-19). Blue dots show the absolute risk predicted from the model across the range of antibody values included in the analysis, adjusting for baseline exposure risk to SARS-CoV-2 infection. Green shaded areas show the confidence interval around the predicted mean probability (green line). $\mathbf{b}, \mathbf{d}, \mathbf{f}, \mathbf{h}$ : Blue shaded areas represent the immune marker density distribution. Green lines show the relative risk of infection among vaccine recipients compared to the MenACWY control arm participants. The green lines are the median relative risk obtained from 10,000 bootstrap samples. Green shaded areas are $95 \%$ bootstrapped confidence intervals for the relative risk. 


\section{Reporting Summary}

Nature Research wishes to improve the reproducibility of the work that we publish. This form provides structure for consistency and transparency in reporting. For further information on Nature Research policies, see our Editorial Policies and the Editorial Policy Checklist.

\section{Statistics}

For all statistical analyses, confirm that the following items are present in the figure legend, table legend, main text, or Methods section.

n/a Confirmed

$\bigotimes$ The exact sample size $(n)$ for each experimental group/condition, given as a discrete number and unit of measurement

$\bigotimes$ A statement on whether measurements were taken from distinct samples or whether the same sample was measured repeatedly

The statistical test(s) used AND whether they are one- or two-sided

Only common tests should be described solely by name; describe more complex techniques in the Methods section.

$\bigotimes$ A description of all covariates tested

Х $\square$ A description of any assumptions or corrections, such as tests of normality and adjustment for multiple comparisons

A full description of the statistical parameters including central tendency (e.g. means) or other basic estimates (e.g. regression coefficient) AND variation (e.g. standard deviation) or associated estimates of uncertainty (e.g. confidence intervals)

For null hypothesis testing, the test statistic (e.g. $F, t, r$ ) with confidence intervals, effect sizes, degrees of freedom and $P$ value noted Give $P$ values as exact values whenever suitable.

\For Bayesian analysis, information on the choice of priors and Markov chain Monte Carlo settings

Х $\square$ For hierarchical and complex designs, identification of the appropriate level for tests and full reporting of outcomes

$\square$ Estimates of effect sizes (e.g. Cohen's $d$, Pearson's $r$ ), indicating how they were calculated

Our web collection on statistics for biologists contains articles on many of the points above.

\section{Software and code}

Policy information about availability of computer code

Data collection RedCap 10.6.13 has been used for data collection (C 2021 Vanderbilt University)

Data analysis Data analysis was done using R version 3.6.1 or later. The GAM was coded using the mgcv package in R. Three knots were used for each GAM, and the smoothing parameter was estimated by generalized cross validation.

For manuscripts utilizing custom algorithms or software that are central to the research but not yet described in published literature, software must be made available to editors and

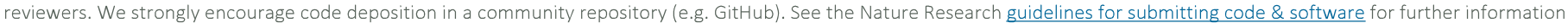

Data

Policy information about availability of data

All manuscripts must include a data availability statement. This statement should provide the following information, where applicable:

- Accession codes, unique identifiers, or web links for publicly available datasets

- A list of figures that have associated raw data

- A description of any restrictions on data availability

Anonymised participant data will be made available when the trials are complete, upon requests directed to the corresponding author. Proposals will be reviewed and approved by the sponsor, investigator, and collaborators on the basis of scientific merit. After approval of a proposal, data can be shared through a secure online platform after signing a data access agreement. All data will be made available for a minimum of 5 years from the end of the trial. 
Please select the one below that is the best fit for your research. If you are not sure, read the appropriate sections before making your selection.

$\bigotimes$ Life sciences $\quad \square$ Behavioural \& social sciences $\quad \square$ Ecological, evolutionary \& environmental sciences

For a reference copy of the document with all sections, see nature.com/documents/nr-reporting-summary-flat.pdf

\section{Life sciences study design}

All studies must disclose on these points even when the disclosure is negative.

Sample size All available data in the trial have been used in this post-hoc analysis on correlates of protection. The sample size for the trial was based on the primary outcomes which are not reported here.

Data exclusions Participants were excluded if they were baseline seropositive or unknown to the SARS-CoV-2 N protein at first vaccination, or had their PB28 visit outside a 14 to 42 day window after the second dose, or were followed up to $<=7$ days after PB28 with no prior evidence of infection, or received mixed schedules in error (details shown in Figure S1). Exclusions were prespecifed in the statistical analysis plan.

\begin{tabular}{l|l} 
Replication & Replication of findings would be impossible as this is a clinical trial of a vaccine against a rapidly mutating pandemic virus in humans
\end{tabular}

Randomization Participants were randomized with full allocation concealment to receive either ChAdOx nCoV-19 vaccine or MenACWY vaccine.

Blinding This is a single-blinded trial where participants were blinded.

\section{Reporting for specific materials, systems and methods}

We require information from authors about some types of materials, experimental systems and methods used in many studies. Here, indicate whether each material, system or method listed is relevant to your study. If you are not sure if a list item applies to your research, read the appropriate section before selecting a response.

\begin{tabular}{|c|c|c|c|}
\hline \multicolumn{2}{|r|}{ Materials \& experimental systems } & \multicolumn{2}{|c|}{ Methods } \\
\hline$n / a$ & Involved in the study & $\mathrm{n} / \mathrm{a}$ & Involved in the study \\
\hline Х & $\square$ Antibodies & Х & ChIP-seq \\
\hline Х & Eukaryotic cell lines & 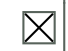 & $\square$ Flow cytometry \\
\hline$\bigotimes$ & $\square$ Palaeontology and archaeology & $\bigotimes$ & $\square$ MRI-based neuroimaging \\
\hline
\end{tabular}

Х $\square$ Animals and other organisms

$\square$ \uman research participants

$\square$ \ Clinical data

$\bigotimes \mid \square$ Dual use research of concern

\section{Human research participants}

Policy information about studies involving human research participants

Population characteristics UK adults aged 18 years old or above; both male and female; generally healthy

Recruitment

Recruitment was done at 19 sites in the UK with a focus on healthcare workers. No selection biases are known of that would affect results.

Ethics oversight

This study was approved in the UK by the Medicines and Healthcare products Regulatory Agency (MHRA), reference 21584/0428/001 0001, and the South-Central Berkshire Research Ethics Committee, reference 20/SC/0179.

All necessary patient/participant informed consent has been obtained and the appropriate institutional forms have been archived.

Note that full information on the approval of the study protocol must also be provided in the manuscript.

\section{Clinical data}

Policy information about clinical studies

All manuscripts should comply with the ICMJE guidelines for publication of clinical research and a completed CONSORT checklist must be included with all submissions.

Clinical trial registration NCT04400838 
Outcomes

Primary symptomatic COVID-19 defined as NAAT+ with at least one of the five COVID symptoms (fever $\geq 37.80 C$; cough; shortness of breath; anosmia orageusia), and asymptomatic SARS-CoV-2 infections defined as a NAAT+ swab with no symptom reported (see more details in Methods and Statistical Plan in supplementary). Outcomes were predefined in the statistical analysis plan. 